SLAC -313

$\mathrm{UC}-34 \mathrm{D}$

(T)

\title{
RESTRICTIONS ON TWO HIGGS DOUBLET MODELS AND CP VIOLATION AT THE UNIFICATION SCALE*
}

\author{
Gregory George Athanasiu \\ Stanford Linear Accelerator Center \\ Stanford University \\ Stanford, California 94305
}

\begin{abstract}
April 1987
Prepared for the Department of Energy

under contract number DE-AC03-76SF00515
\end{abstract}

Printed in the United States of America. Available from the National Technical Information Service, U.S. Department of Commerce, 5285 Port Royal Road, Springfield, Virginia 22161. Price: Printed Copy A05, Microfiche A01.

* Ph.D. Dissertation 


\section{Table of Contents}

page

INTRODUCTION $\ldots \ldots \ldots \ldots \ldots \ldots \ldots$

PART I: Bounds on Charged Higgs Properties From CP violation in the Kaon system

Chapter 1. Introduction $\ldots \ldots \ldots \ldots$

Chapter 2. Bounds from $\epsilon \ldots \ldots \ldots \ldots$

Chapter 3. Consequences for $\epsilon^{\prime} \ldots \ldots \ldots \ldots$

References ......................12

PART II: Restrictions on Two Higgs Doublet Models from Heavy Quark Systems

Chapter 1. Introduction $\ldots \ldots \ldots \ldots \ldots$

Chapter 2. Limits from $B^{0}-\bar{B}^{0}$ Mixing $\ldots \ldots \ldots \ldots$

Chapter 3. Limits from $t \bar{t}$ Spectroscopy _. . . . . . . . . . 28

Chapter 4. Conclusion $\ldots \ldots \ldots \ldots \ldots \ldots$

References ........................ . . 40

PART III: CP violation at the Unification Scale

Chapter 1. Introduction $\ldots \ldots \ldots \ldots \ldots$ 
Chapter 2. Renormalization Group Equations in the Standard Model . . . 59

Chapter 3. Extension to Two Higgs Doublets and Susy . . . . . . 61

Chapter 4.Baryogenesis ...................... 63

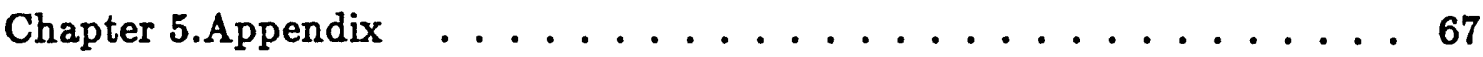

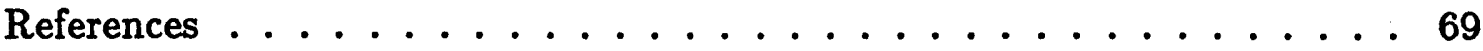




\section{INTRODUCTION}

In Part I we examine bounds from CP violation in the neutral $K$ system on charged Higgs masses and couplings in models with two Higgs doublets. While CP violation is still due only to a non-zero phase in the Kobayashi-Maskawa matrix, there are additional short-distance contributions involving charged Higgs exchange rather than $\mathrm{W}$ boson exchange. By having $\mathrm{CP}$ violation in the mass matrix, but not in the kaon to two pions decay amplitude, largely due to Higgs exchange, it is possible to obtain a small value of $\epsilon^{\prime} / \epsilon$.

In Part II we obtain bounds on charged-Higgs-boson masses and couplings in two Higgs doublet models from their effects on neutral- $B$-meson mixing. The bounds are comparable to those obtained with additional assumptions from the neutral- $K$-system. Neutral-Higgs-boson effects on the spectrum and wave functions of $t \bar{t}$ bound states are examined in the same model. In the future they could lead to restrictions on, or discovery of, the corresponding neutral Higgs bosons if they have relatively low masses and enhanced couplings.

Finally, in Part III, the three generation phase invariant measure of CP violation is shown to satisfy a simple and solvable renormalization group equation. Its value falls by four to eight orders of magnitude between the weak and grand unification scales in the standard model, as well as in its two Higgs and supersym-

metric extensions. Such a small value of $\mathrm{CP}$ violation at the grand unification scale can pose a problem for baryogenesis; this is avoided if there are heavy quarks with masses close to their fixed points. 


\section{PART I}

\section{BOUNDS ON CHARGED HIGGS PROPERTIES FROM CP VIOLATION}

\section{Introduction}

With the emergence of the standard model and its origin for $C P$ violation in a phase within the Kobayashi-Maskawa ${ }^{[1]}(\mathrm{K}-\mathrm{M})$ matrix describing the weak couplings of quarks, it is of great importance to test whether this is the correct explanation of $C P$ violation by delineating its consequences for as many specific cases as possible and by subjecting them to experimental test. Thus we have, for example, the attempts ${ }^{[2]}$ to calculate the parameters $\epsilon$ and $\epsilon^{\prime}$ of $C P$ violation in the neutral $K$ system in terms of the elements of the K-M matrix plus values of matrix elements of relevant operators and the recent experiments ${ }^{[3]}$ to measure $\epsilon^{\prime} / \epsilon$ with high accuracy.

In a different vein, but also very much related to the standard model, there is much interest in the Higgs sector. The neutral Higgs boson remains as the key undiscovered particle of that model, and there is also considerable speculation on whether the Higgs sector should be enlarged or even totally replaced by a dynamics. These latter possibilities affect the question of $C P$ violation since the introduction of additional Higgs generates at a minimum extra diagrams involving Higgs exchange to be considered along with those involving $W$ exchange. At most, in some models with three or more Higgs doublets, ${ }^{(4)}$ the Higgs sector can become the sole source of $C P$ violation.

Here we shall be interested in the extension of the minimal (standard) model to the case of two Higgs doublets rather than one, although many of our results 
can be generalized easily beyond the case of two doublets. We are concerned with what restrictions the observed $C P$ violation in the neutral $K$ system places on the couplings and masses of the charged Higgs bosons in such a theory. The restrictions which follow from the tiny $K_{L}^{0}-K_{S}^{0}$ mass difference have already been studied, ${ }^{[s]}$ but $C P$ violating effects are even smaller (by $\sim 10^{-3}$ ) and emphasize different $\mathrm{K}-\mathrm{M}$ angles and different quarks. Correspondingly we get even more sensitive bounds than obtained from the mass difference if we adopt the same kind of criteria.

Looked at another way, introducing additional Higgs bosons and therefore additional diagrams gives us more freedom in attempting to explain present observations. We shall also take this viewpoint and will find that it is possible for the Higgs exchange contribution to be the primary source of $C P$ violation in the neutral $K$ mass matrix (i.e., the parameter $\epsilon$ ), while not being the dominant source of $C P$ violation in $K$ decay (i.e., the parameter $\epsilon^{\prime}$ ). Therefore, if the standard model runs into trouble accounting simultaneously for the values of both $\epsilon$ and $\epsilon^{\prime}$, the introduction of another Higgs doublet with resulting heavy charged Higgs bosons could be a relatively "cheap" extension of the standard model that "decouples" the source of $\epsilon$ and $\epsilon$ ' and allows for consistency with experiment. 


\section{Bounds from $\epsilon$}

Let us first follow the path toward achieving bounds that Abbott, Sikivie, and $W$ ise ${ }^{(5)}$ applied to the real part of the mass matrix. Namely, we adopt the philosophy that the imaginary part of the $K^{0}-\bar{K}^{0}$ mass matrix element (proportional to $\epsilon$ ) is "understood" as arising largely from the short distance contributions associated with the box diagram involving two $W$ 's and two heavy quarks. Correspondingly, the contribution from exchange of two Higgs bosons and from a $W$ and a Higgs boson is assumed to be smaller than the standard one involving two $W$ 's, i.e.

$$
\epsilon_{H B}+\epsilon_{B W}<\epsilon_{W W}
$$

in order not to "spoil" the assumed approximate agreement with experiment of $\epsilon_{w w} \cdot$

In a model with extra Higgs doublets we want to preserve the property that there are no flavor changing neutral currents at tree level. This can be accomplished ${ }^{[6]}$ by having one neutral Higgs field coupled to charge $2 / 3$ quarks and another Higgs field coupled to charge $-1 / 3$ quarks. In this case the coupling of the physical charged Higgs bosons is given by ${ }^{|s|}$

$$
\mathcal{L}_{\text {int }}=\frac{g \phi^{+}}{2 \sqrt{2} M_{W}} \bar{U}\left[\frac{\xi}{\eta} M_{u} K\left(1-\gamma_{5}\right)+\frac{\eta}{\xi} K M_{d}\left(1+\gamma_{5}\right)\right] D+\text { H.c. }
$$

where $\eta$ and $\xi$ are the vacuum expectation values of the Higgs fields coupled to charge $2 / 3$ and $-1 / 3$ quarks, respectively. The $3 \times 3$ matrix $K$ is the K-M matrix ${ }^{[1]}$ and $M_{u}$ and $M_{d}$ are diagonal mass matrices for charge $2 / 3$ and $-1 / 3$ quarks $U$ and $D$, respectively. 
Alternatively, one can avoid flavor changing neutral currents by having just one of the two Higgs doublets couple to quarks. ${ }^{[7]}$ In this case the neutral Higgs couplings are diagonalized along with the mass matrix and the charged Higgs couplings are given by ${ }^{[5,7]}$

$$
\mathcal{L}_{\text {int }}=\frac{g}{2 \sqrt{2} M_{W}} \phi^{+} \bar{U}\left[\frac{\xi}{\eta} M_{u} K\left(1-\gamma_{5}\right)-\frac{\xi}{\eta} M_{d}\left(1+\gamma_{5}\right)\right] D+\text { H.c. . }
$$

Since for the heavy quarks the mass of the charge $2 / 3$ quarks is much greater than that of the charge $-1 / 3$ quarks in the same generation, it is the term proportional to $(\xi / \eta) M_{u}$ in either Eq. (2) or (3) which gives the best possibility of significant Higgs couplings between light and heavy quarks. Therefore, from here on, we concentrate only on this term with $\xi / \eta>1$.

The imaginary part of the $\Delta S=2$ effective Hamiltonian responsible for $K^{0}-\bar{K}^{0}$ mixing then has the form

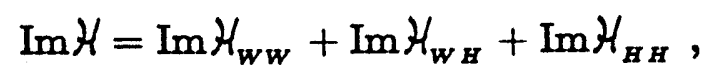

where $^{[8]}$

$$
\begin{aligned}
\operatorname{Im} \mathcal{H}_{W W}= & \frac{g^{4} s_{1}^{2} s_{2} s_{2} s_{\delta}}{2^{8} \pi^{2} M_{W}^{4}}\left\{-m_{c}^{2}+m_{c}^{2} \ln \frac{m_{t}^{2}}{m_{c}^{2}}+s_{2}\left(s_{2}+s_{3} c_{\delta}\right) m_{t}^{2}\right\} \\
& \times\left[\bar{s} \gamma_{\mu}\left(1-\gamma_{5}\right) d\right]\left[\bar{s} \gamma^{\mu}\left(1-\gamma_{5}\right) d\right], \\
\operatorname{Im} \mathcal{H}_{W H}= & \frac{g^{4} s_{1}^{2} s_{2} s_{3} s_{\delta}}{2^{6} M_{W}^{4}}\left(\frac{\xi}{\eta}\right)^{2}\left\{-m_{c}^{4}\left(8 M_{W}^{2} I_{2}\left(m_{c}\right)+2 I_{3}\left(m_{c}\right)\right)\right.
\end{aligned}
$$




$$
\begin{aligned}
& +m_{c}^{2} m_{t}^{2}\left(8 M_{W}^{2} I_{5}+2 I_{6}\right) \\
& +s_{2}\left(s_{2}+s_{3} c_{\delta}\right) m_{t}^{4}\left(8 M_{W}^{2} I_{2}\left(m_{t}\right)+2 I_{3}\left(m_{t}\right)\right\} \\
& \times\left[\bar{s} \gamma_{\mu}\left(1-\gamma_{5}\right) d\right]\left[\bar{s} \gamma^{\mu}\left(1-\gamma_{5}\right) d\right],
\end{aligned}
$$

and

$$
\begin{aligned}
\operatorname{Im} \mathcal{K}_{H B}= & \frac{g^{4} s_{1}^{2} s_{2} s_{3} s_{\delta}}{2^{6} M_{W}^{4}}\left(\frac{\xi}{\eta}\right)^{4} \\
& \times\left\{-m_{c}^{4} I_{1}\left(m_{c}\right)+m_{c}^{2} m_{t}^{2} I_{4}+s_{2}\left(s_{2}+s_{3} c_{\delta}\right) m_{t}^{4} I_{1}\left(m_{t}\right)\right\} \\
& \times\left[\bar{s} \gamma_{\mu}\left(1-\gamma_{5}\right) d\right]\left[\bar{s} \gamma^{\mu}\left(1-\gamma_{5}\right) d\right]
\end{aligned}
$$

Here the integrals $I_{1}, \ldots, I_{6}$ are defined in Abbott, Sikivie and Wise. ${ }^{|5|}$ Knowing that the K-M angles $\theta_{1}, \theta_{2}$, and $\theta_{3}$ are all small, ${ }^{[8]}$ we have used the very good approximation that $\cos \theta_{i}=1$. (But the $C P$ violating phase $\delta$ may well be large, so we keep both $\cos \delta=c_{\delta}$ and $\sin \delta=s_{\delta}$.) As the subscripts imply, $\mathcal{K}_{w w}, \mathcal{H}_{w H}$ and $\mathcal{H}_{H H}$ arise from the short distance box graph involving exchange respectively of two $W$ 's (the standard contribution ${ }^{[0]}$ ), a $W$ and a charged Higgs boson and two charged Higgs bosons. The imaginary part of $\mathcal{H}$ and hence $C P$ violation in the neutral $K$ system arise entirely because of a non-zero K-M phase $\delta$ in each 
term of Eq. (4). When $\delta=0$ there is no $C P$ violation inherent in the Higgs sector itself, as there may be in models with three or more Higgs doublets.|[

We now impose the condition in Eq. (1). Since $\operatorname{Im} \not{H} \propto \epsilon$ and the effective Hamiltonians in Eqs. (5a), (5b) and (5c) all involve the same four-quark operator, the matrix element of that operator cancels out of the resulting equation along with the weak coupling $g$ and the common factor $s_{1}^{2} s_{2} s_{3} s_{\delta}$. Inasmuch as we are interested in bounding $\xi / \eta$ when $M_{H}^{2} \gg m_{t}^{2}$, and since $m_{t} \gg m_{c}$, a good first approximation to the resulting inequality is obtained by only keeping the term proportional to $m_{t}^{4}(\xi / \eta)^{4}$ in Eq. (5c) and that involving $m_{t}^{2}$ in Eq. (5a). This results in

$$
\left(\frac{\xi}{\eta}\right)^{2}<2\left(\frac{M_{H}}{m_{t}}\right)
$$

when we use the expression ${ }^{|s|}$ for $I_{1}(m)=\left(16 \pi^{2} M_{H}^{2}\right)^{-1}$ valid to $O\left(m^{2} / M_{H}^{4}\right)$.

The exact bound following from the full expression, a quadratic in $(\xi / \eta)^{2}$, is not much harder to compute. While the factor $g^{4} s_{1}^{2} s_{2} s_{3} s_{\delta}$ still cancels out, there is now a dependence on the K-M angles through the quantity $s_{2}\left(s_{2}+s_{3} c_{\delta}\right)$ which enters Eqs. (5) in the terms arising purely from $t$ quark exchange. An example of the bound on $(\xi / \eta)^{2}$ for a typical value ${ }^{[10]}$ of $s_{2}\left(s_{2}+s_{3} c_{\delta}\right)=2.5 \times 10^{-3}$ and for $m_{t}=45 \mathrm{GeV}$ is shown in Fig. 1. Varying $s_{2}\left(s_{2}+s_{3} c_{\delta}\right)$ from $1 \times 10^{-3}$ to $5 \times 10^{-3}$ changes this upper bound by $\sim 30 \%$ (downward). The bound (Eq. (6)) obtained by keeping only the leading terms in $m_{t}$ (the dashed line in Fig. 1) is obviously a good approximation to the exact bound (the solid curve).

The upper bound on $(\xi / \eta)^{2}$ obtained here is much stronger (by a factor of $\sim 20)$ than that ${ }^{|5|}$ obtained from the real part of the $K^{0}-\bar{K}^{0}$ mass matrix under 
analogous assumptions on the relative size of the Higgs and $W$ contributions. For example, instead of ${ }^{|\xi|}(\xi / \eta)^{2} \lessgtr 200$ at $M_{H}=150 \mathrm{GeV}$, we have $(\xi / \eta)^{2} \lessgtr 10$. Even for charged Higgs bosons with masses of a sizeable fraction of a TeV, Fig. 1 implies $(\xi / \eta)^{2} \lessgtr 25$. Thus within the constraint imposed by adopting Eq. (1), enhancement ${ }^{[11]}$ of the Higgs coupling to quarks by more than a factor $\xi / \eta \sim 5$ is ruled out for "reasonable" charged Higgs masses.

We now change our viewpoint and adopt an alternative philosophy, allowing the diagrams involving charged Higgs exchange rather than $W$ exchange to be the main source of $C P$ violation in the neutral $K$ mass matrix. We replace Eq. (1) by

$$
\epsilon_{W w}+\epsilon_{W B}+\epsilon_{B H}=\epsilon
$$

and use the experimental value ${ }^{[8]}$ of $2.27 \times 10^{-3}$ on the right-hand side. Depending on the values of the K-M angles, Higgs parameters, etc., either the terms involving Higgs exchange or those involving $W$ exchange on the left-hand side of Eq. (7) could be the primary source of $\epsilon$.

In particular, the $K^{0}-\bar{K}^{0}$ matrix element of $\left[\bar{s} \gamma_{\mu}\left(1-\gamma_{5}\right) d\right]\left[\bar{s} \gamma^{\mu}\left(1-\gamma_{5}\right) d\right]$ no longer cancels out, nor does the characteristic combination of mixing angles $s_{1}^{2} s_{2} s_{3} s_{\delta}$. Defining in a conventional way the parameter $B$ as the ratio of the actual matrix element to its vacuum-insertion value, the factor $B s_{1}^{2} s_{2} s_{3} s_{\delta}$ is common to all terms on the left-hand side of Eq. (7). The resulting equation is a quadratic in $(\xi / \eta)^{2}$ whose solutions we can parametrize in terms of $B s_{1}^{2} s_{2} s_{3} s_{\delta}$, $M_{H} / m_{t}$ and $s_{2}\left(s_{2}+s_{3} c_{\delta}\right)$.

At one extreme we have solutions where, as before, $\epsilon_{w w} \gg \epsilon_{W H}+\epsilon_{H H}$. 
The relevant domain of parameters may be obtained by noting that there is a constraint following from the condition that $(\xi / \eta)^{2} \geq 0$ for the solutions of Eq. (7), treated as a quadratic equation in $(\xi / \eta)^{2}$. For $s_{2}\left(s_{2}+s_{3} c_{\delta}\right)=2.5 \times 10^{-3}$, we find ${ }^{[12]} B s_{1}^{2} s_{2} s_{3} s_{\delta} \leq 2.14 \times 10^{-5}$ independent of $M_{H} / m_{t}$, with the equality holding when there is no Higgs contribution in Eq. (7). As shown by the solid curve in Fig. 2, for a value of $B s_{1}^{2} s_{2} s_{3} s_{6}=2.1 \times 10^{-5}$ (just lightly less than the bound) the solutions to Eq. (7) involve relatively small values of $(\xi / \eta)^{2}$ and have only a mild dependence on $M_{H} / m_{t}$. In this particular example $\epsilon_{w w}$ is the source of $98 \%$ of $\epsilon$.

At the other extreme, when $B s_{1}^{2} s_{2} s_{3} s_{\delta}$ is much smaller than its maximum, one has contributions from Higgs exchange as the dominant source of $\epsilon$. When for example, $B s_{1}^{2} s_{2} s_{3} s_{\delta}=1 \times 10^{-6}, \epsilon_{w w}$ supplies only $5 \%$ of $\epsilon$ and $(\xi / \eta)^{2}$ is large and depends almost linearly on $M_{H} / m_{t}$ (as shown by the dashed curve in Fig. 2.). Thus the short-distance contribution due to Higgs exchange could be the dominant contribution to $C P$ violation in the neutral $K$ mass matrix. Associated with this situation is a small value of $B s_{1}^{2} s_{2} s_{3} s_{\delta}$ (as compared with its value when the usual $W$ exchange contribution is the primary source of $\epsilon$ ).

\section{Consequences on $\epsilon^{\prime}$}

At the same time we may consider what happens to the other parameter of $C P$ violation in the neutral $K$ system, $\epsilon^{\prime}$. This measures $C P$ violation in the $K \rightarrow \pi \pi$ decay amplitude and originates ${ }^{[13]}$ primarily from so-called "penguin" diagrams. Here also we will have an additional diagram obtained by replacing $W$ exchange with charged Higgs exchange. Their amplitudes can be related ${ }^{|8|}$ by a 
Fierz transformation and their relative contributions to $\epsilon^{\prime}$ are in the ratio

$$
\frac{\epsilon_{H}^{\prime}}{\epsilon_{W}^{\prime}} \approx \frac{A_{H}^{\text {Penguin }}}{A_{W}^{\text {Penguin }}} \approx-\frac{1}{2}\left(\frac{\xi}{\eta}\right)^{2} \frac{m_{t}^{2}}{M_{H}^{2}} \frac{\ln \left(M_{H}^{2} / m_{t}^{2}\right)}{\ln \left(m_{t}^{2} / m_{c}^{2}\right)}
$$

Comparing this to the leading (in $m_{t}$ ) contributions to $\epsilon$ :

$$
\frac{\epsilon_{H H}}{\epsilon_{w W}} \approx\left[\frac{1}{2}\left(\frac{\xi}{\eta}\right)^{2} \frac{m_{t}}{M_{H}}\right]^{2}
$$

we see that aside from logarithms, the ratio of the Higgs contribution to the $W$ contribution in $\epsilon^{\prime}$ is down by a factor $m_{t} / M_{H}$ as compared to the situation in $\epsilon^{1 / 2}$. Therefore if $(\xi / \eta)^{2}\left(m_{t} / M_{H}\right)$ is $O(1)$ or less, as it is when $\epsilon_{W W}>\epsilon_{W H}+\epsilon_{H B}$, then the Higgs exchange contribution to $\epsilon^{\prime}$ is an order of magnitude or more smaller than that of $W$ exchange in the domain $m_{t}^{2} / M_{H}^{2} \ll 1$ that we are considering. But even when $(\xi / \eta)^{2}\left(m_{t} / M_{W}\right)$ is large (say $\left.\sim 10\right)$ and Higgs exchange gives by far the dominant contribution to $\epsilon$, the contribution from Higgs exchange to $\epsilon^{\prime}$ is at most comparable in magnitude to that of $W$ exchange. ${ }^{[14]}$

Thus even when the Higgs exchange contribution dominates $\epsilon$, we still have $\epsilon^{\prime} \approx \epsilon_{W}^{\prime}$. But the absolute magnitude of $\epsilon_{W}^{\prime}$ is proportional to a product of a $K \rightarrow \pi \pi$ matrix element of the penguin operator and of its coefficient, involving the overall factor $s_{1}^{2} s_{2} s_{3} s_{\delta}$. When we go from the situation where $W$ exchange contributions dominate $\epsilon$ to that where Higgs exchange contributions dominate, everything in the calculation of $\epsilon_{W}^{\prime}$ remains the same except that $s_{1}^{2} s_{2} s_{3} s_{\delta}$ decreases (proportionally) as $\epsilon_{w w} / \epsilon$ decreases: by "tuning" up the portion of $\epsilon$ to be accounted for by Higgs exchange contributions rather than the standard $W$ exchange contributions, we can reduce ${ }^{[14]}$ the predicted value of $\epsilon^{\prime}$. Therefore, 
by extending the minimal model through the introduction of a second Higgs doublet involving heavy charged Higgs bosons with enhanced couplings, one could accommodate a very small value of $\epsilon^{\prime} / \epsilon$. 


\section{FIGURE CAPTIONS}

1) Upper bound on $(\xi / \eta)^{2}$ as a function of $M_{H} / m_{t}$ following from the condition $\epsilon_{H B}+\epsilon_{W H}<\epsilon_{W W}$. The resulting approximate bound in Eq. (6) is shown (dashed line), as well as the exact bound (solid curve) for $s_{2}\left(s_{2}+\right.$ $\left.s_{3} c_{\delta}\right)=2.5 \times 10^{-3}, m_{c}=1.5 \mathrm{GeV}, m_{t}=45 \mathrm{GeV}$.

2) Value of $(\xi / \eta)^{2}$ as a function of $M_{H} / m_{t}$ needed to satisfy $\epsilon_{W W}+\epsilon_{W B}+\epsilon_{B H}=$ $\epsilon$ when $B s_{1}^{2} s_{2} s_{3} s_{\delta}$ equals $2.1 \times 10^{-5}$ (solid curve), $8 \times 10^{-6}$ (dotted curve), and $1 \times 10^{-6}$ (dashed curve). The parameters $m_{c}=1.5 \mathrm{GeV}, m_{t}=45 \mathrm{GeV}$ and $s_{2}\left(s_{2}+s_{3} c_{6}\right)=2.5 \times 10^{-3}$.

\section{REFERENCES}

1. M. Kobayashi and T. Maskawa, Prog. Theo. Phys. $\underline{49}, 652$ (1973).

2. The theoretical situation is reviewed by M. B. Wise, talk at the Topical Conference of the 1984 SLAC Summer Institute on Particle Physics, July 23-August 3, 1984 and Caltech preprint CALT-689-1179, 1984 (unpublished).

3. The experimental situation is reviewed by J. Cronin, talk at the Topical Conference of the 1984 SLAC Summer Institute on Particle Physics, July 23-August 3, 1984 (unpublished).

4. S. Weinberg, Phys. Rev. Letters $\underline{37}, 657$ (1976); T. D. Lee, Phys. Rev. D8, 1226 (1973) and Phys. Rep. 9C, 143 (1974); P. Sikivie, Phys. Lett. $\underline{\text { 65B, }}$ 141 (1976). 
5. L. F. Abbott, P. Sikivie, and M. B. Wise, Phys. Rev. D21, 1393 (1980). These authors consider the QCD corrections to the Higgs exchange contributions as well, but conclude that the effects on the phenomenological implications are small.

6. S. L. Glashow and S. Weinberg, Phys. Rev. D15, 1958 (1977).

7. H. E. Haber, G. L. Kane and T. Sterling, Nucl. Phys. B161, 493 (1979).

8. Particle Data Group, Rev. Mod. Phys. $\underline{56}$, No. 2, Part 11, S296 (1984).

9. M. K. Gaillard and B. W. Lee, Phys. Rev. D10, 897 (1974); J. Ellis, M. K. Gaillard and D. V. Nanopoulos, Nucl. Phys. B109, 213 (1976).

10. From the constraints on the K-M angles following from the $b$ lifetime and the upper bound on $(b \rightarrow u) /(b \rightarrow c)$ given in Ref. 8, we take $s_{2}\left(s_{2}+s_{3} c_{\delta}\right)$ to be in the range $1 \times 10^{-3}$ to $5 \times 10^{-3}$ with a "typical" value of $2.5 \times 10^{-3}$.

11. Other constraints on this enhancement have been considered by M. Sher and D. Silverman Phys.Rev. D31 , 95 (1985). See also the summary on nonstandard Higgs bosons by P. Langacker et al., University of Pennsylvania preprint UPR-0269T, 1984.Published in the Proceedings of the 1984 Summer Study on the Design and Utilization of the Superconducting Super Collider, Snowmass, Colorado,p.771, June 23-July 13, 1984.

12. For $s_{2}\left(s_{2}+s_{3} c_{\delta}\right)=1 \times 10^{-3}$ and $5 \times 10^{-3}$, we find $B s_{1}^{2} s_{2} s_{3} s_{\delta} \leq 2.57 \times 10^{-5}$ and $1.67 \times 10^{-5}$, respectively.

13. F. J. Gilman and M. B. Wise, Phys. Lett. $\underline{83 B}, 83$ (1979) and Phys. Rev. D20, 2392 (1979). 
14. From Eq. (8) we note that the minus sign on the right-hand side makes $\epsilon^{\prime}=\epsilon_{W}^{\prime}+\epsilon_{H}^{\prime}$ smaller in magnitude than $\epsilon_{W}^{\prime}$. 


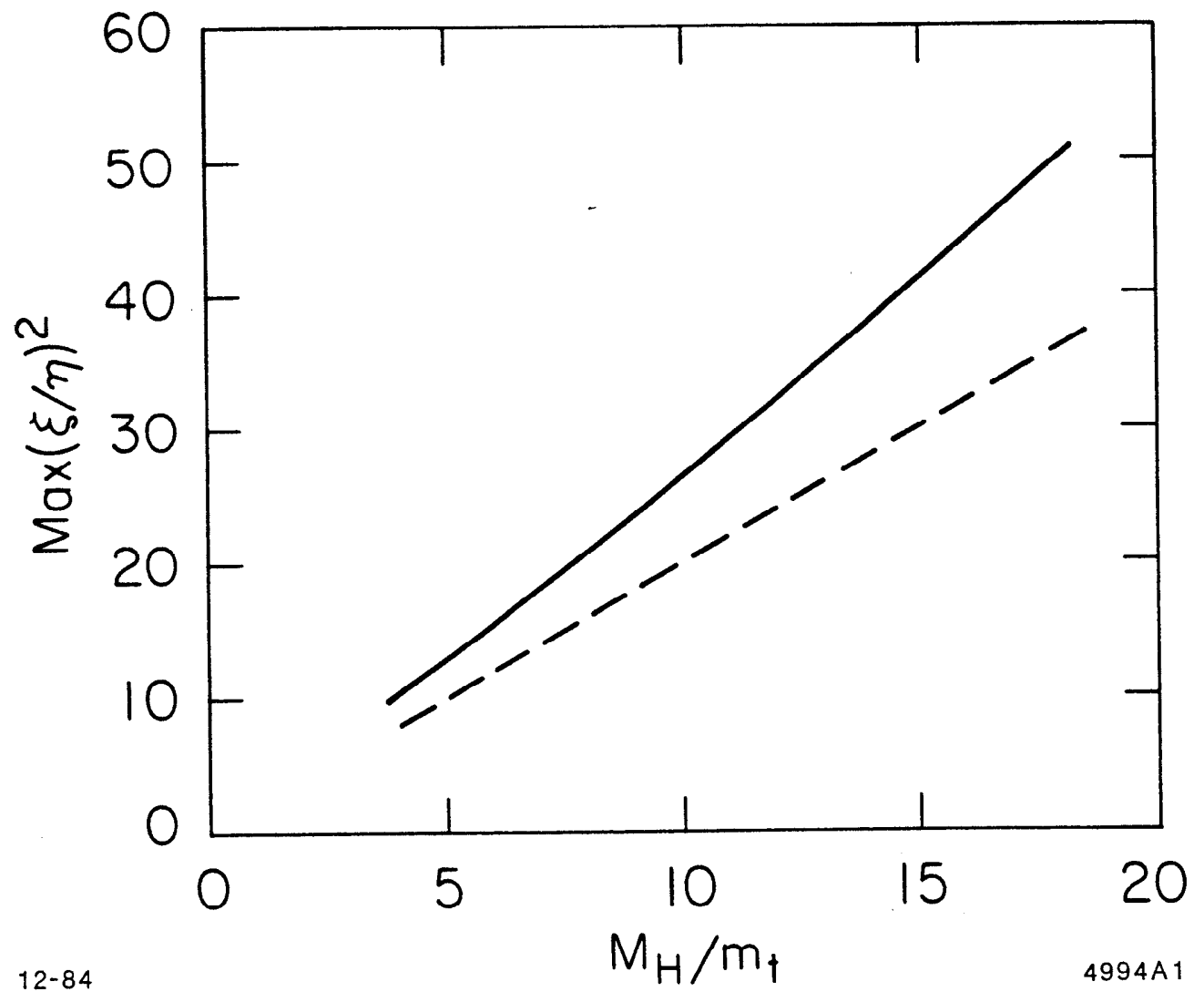

FIGURE 1 


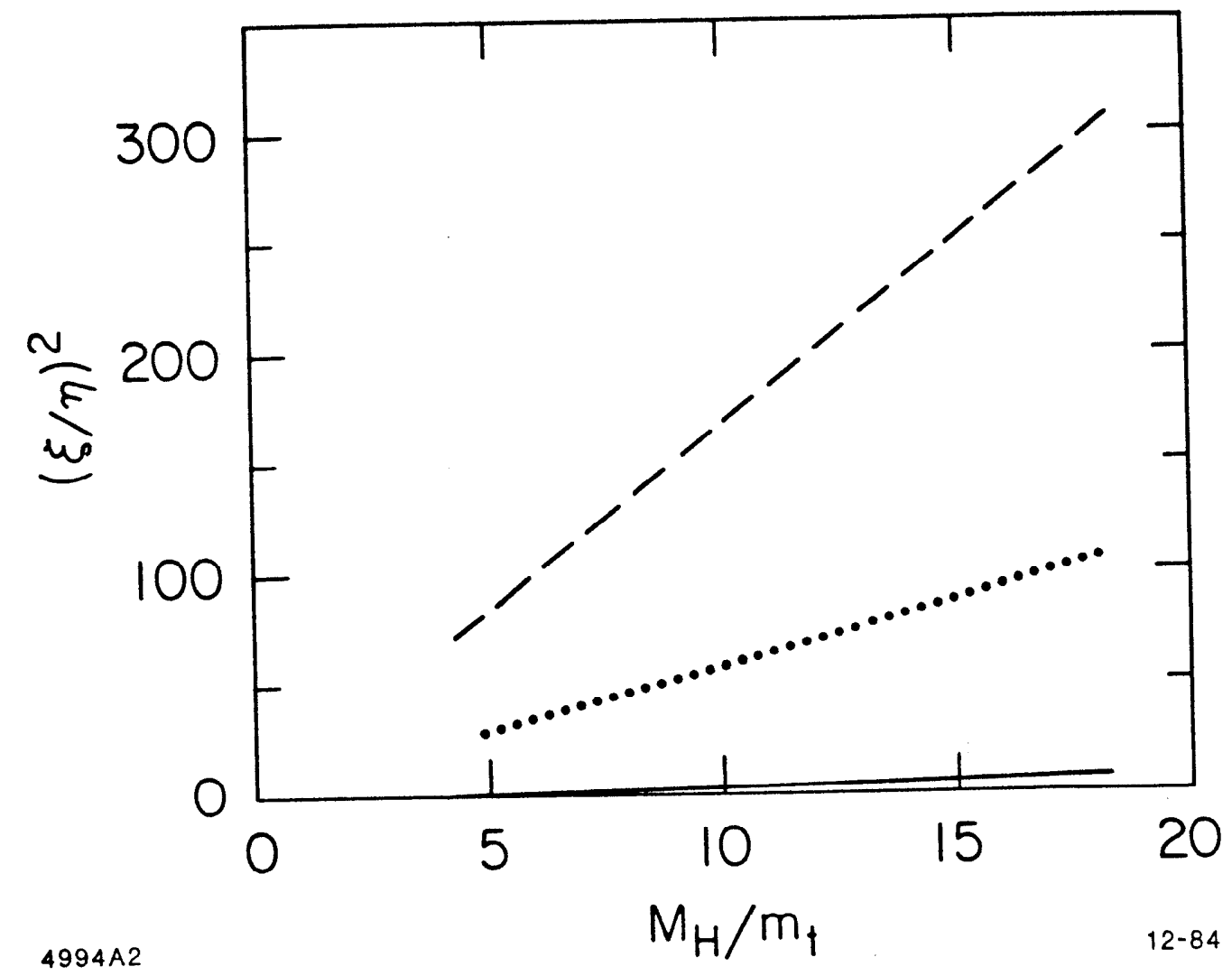

FIGURE 2 


\section{PART II \\ RESTRICTIONS ON TWO HIGGS DOUBLET MODELS \\ FROM HEAVY QUARK SYSTEMS}

\section{Introduction}

While even the single neutral physical Higgs boson of the standard model ${ }^{[1]}$ is yet to be found, there is considerable speculation that the Higgs sector is to be enlarged, ${ }^{[2]}$ if not to be replaced altogether by dynamically generated states which are only one manifestation of a whole spectrum of particles due to an additional kind of strong interaction. ${ }^{|s|}$ At a less dramatic level, currently interesting models involving left-right symmetric gauge theories, ${ }^{[A]}$ or supersymmetry, for example, call for an enlargement of the Higgs sector to involve at least two Higgs doublets.

In a theory with two Higgs doublets we gain four more physical bosons, two charged and two neutral. At the same time there is an additional parameter in a second vacuum expectation value, or, more conveniently, a ratio of vacuum expectation values if we fix one appropriate combination to be that of the standard model. Tuning this ratio of vacuum expectation values allows one to enhance (or suppress) the strength of the physical Higgs couplings and thereby to increase (or decrease) the size of the effects these additional bosons have on various processes.

Abbott, Sikivie, and Wise ${ }^{[6]}$ showed that useful bounds on the enhancement of the couplings of the charged Higgs bosons in such a model could be set by considering their effect on the $K_{S}^{0}-K_{L}^{0}$ mass difference. Because the charged Higgs bosons couple proportionally to the mass of the fermion and their contributions are not subject to a GIM cancellation, ${ }^{[1]}$ they potentially give a large 
short-distance contribution to this mass difference through their presence together with heavy quarks in the relevant one loop diagrams. In the case of the $K_{S}^{0}-K_{L}^{0}$ mass difference it is the charm quark which is responsible for most of the short-distance contribution and therefore the charm quark mass which enters the bound derived in this manner.

More recently, the bounds derivable from the imaginary, i.e. CP violating, part of the neutral $K$ mass matrix have been investigated. ${ }^{[7]}$ Here the top quark plays a dominant role, and the resulting bounds are much stronger than those of Abbott, Sikivie and Wise, ${ }^{[6]}$ if the assumption is again made that the shortdistance contribution due to diagrams involving Higgs exchange is less than that due to $W$ exchange. However, it is altogether possible to contemplate dropping this last requirement, in which case the Higgs exchange diagrams could become the primary source of $C P$ violation in the neutral $K$ mass matrix, and a fairly large range of Higgs masses and couplings is opened up.

In this paper we obtain the bounds on masses and couplings of charged Higgs bosons in a two doublet model that follow from their effect on neutral B meson mixing, i.e. the $B_{S}^{0}-B_{L}^{0}$ mass difference. Again, virtual $t$ quarks play the dominant role. However, in this case we obtain useful bounds independent of assumptions on the relative magnitude of the short distance contributions. Furthermore, as shown in Section II, from the experimental constraints on $B^{0}-\bar{B}^{0}$ mixing we obtain quite stringent bounds. They are comparable to the best bounds ${ }^{[7]}$ obtained previously in the neutral $K$ system with the additional assumption discussed above on the relative magnitude of Higgs and $W$ contributions. 
In Section III we turn our attention to the neutral Higgs particles. We investigate in some detail a subject looked at previously: the effect of neutral Higgs boson exchange on the spectrum and wavefunctions of toponium. ${ }^{[8]}$ We consider in particular the problem of unambiguously distinguishing the effects of the Higgs boson from the effects of different, but theoretically acceptable, potentials. The net restrictions following from having considered both charged and neutral Higgs bosons are summarized in Section IV.

\section{Limits from $B^{0}-\bar{B}^{0}$ mixing}

As we have mentioned, many modifications and extensions of the standard model require extra Higgs multiplets. We shall be considering here the specific model with two Higgs doublets, although much of what we do can easily be extended to more drastic additions to the standard model.

In any model with extra Higgs doublets, care must be taken to preserve the property that there be no flavor changing neutral currents at tree level. This can be accomplished in two ways. First, we can have one neutral Higgs field coupled to charge $\frac{2}{3}$ quarks and another Higgs field coupled to charge $-\frac{1}{3}$ quarks. ${ }^{|0|}$ In this case the coupling of the physical charged bosons is given by ${ }^{[0]}$

$$
\mathcal{L}_{\text {int }}=\frac{g \phi^{+}}{2 \sqrt{2} M_{W}} \bar{U}\left[\frac{\xi}{\eta} M_{u} K\left(1-\gamma_{5}\right)+\frac{\eta}{\xi} K M_{d}\left(1+\gamma_{5}\right)\right] D+H . c .
$$

where $\eta$ and $\xi$ are the vacuum expectation values of the unmixed Higgs fields coupled to charge $\frac{2}{3}$ and $-\frac{1}{3}$ quarks, respectively. The $3 \times 3$ matrix $K$ is the Kobayashi-Maskawa(K-M) matrix, ${ }^{[10]}$ and $M_{u}$ and $M_{d}$ are diagonal mass matrices for the three charge $\frac{2}{3}$ and $-\frac{1}{3}$ quarks $U$ and $D$, respectively. 
Second, we can avoid flavor changing neutral currents by having just one Higgs doublet couple to quarks, ${ }^{[1]}$ as in the standard model. In this case the neutral Higgs couplings are diagonalized along with the mass matrix and the charged Higgs couplings are given by ${ }^{[6,11]}$

$$
\mathcal{L}_{\text {int }}=\frac{g \phi^{+}}{2 \sqrt{2} M_{W}} \bar{U}\left[\frac{\xi}{\eta} M_{u} K\left(1-\gamma_{5}\right)-\frac{\xi}{\eta} K M_{d}\left(1+\gamma_{5}\right)\right] D+H . c .,
$$

Since for the second and third generations the mass of the charge $\frac{2}{3}$ quarks is much greater than that of the charge $-\frac{1}{3}$ quarks in the same generation, it is the term proportional to $\left(\frac{\xi}{\eta}\right) M_{u}$ in either Eq. (2.1) or (2.2) which gives the possibility of a significant enhancement of the Higgs couplings between light and heavy quarks. Therefore it is this term upon which we have the best possibility of imposing bounds from experimental constraints. Henceforth we shall concentrate on its effects on physical quantities, thereby bounding $\frac{\xi}{\eta}$.

The first bounds on $\frac{\xi}{\eta}$ in models with two Higgs doublets came ${ }^{|6|}$ from looking at the $K_{S}-K_{L}$ mass difference and in particular the short-distance contributions to this mass difference arising from the box diagrams with heavy quarks and $W$ 's or Higgs bosons running around the internal loop (see Fig. 1). The usual contribution involving $W$ 's leads to an effective operator with a coefficient which because of the GIM cancellation ${ }^{[1]}$ behaves as $G_{F}^{2} m_{q}^{2}$, aside from factors coming from the K-M matrix. That involving Higgs bosons on the other hand, has no GIM cancellation and behaves as $G_{F}^{2}\left(\frac{\xi}{\eta}\right)^{4} \frac{m_{q}^{4}}{M_{H}^{2}}$ aside from the same K-M factors. Thus, if we impose the condition that the short-distance contribution from the diagrams involving Higgs bosons be less than that due to diagrams involving $W$ 's, we will characteristically arrive at bounds of the form $\left(\frac{\xi}{\eta}\right)^{2}<O\left(\frac{M_{H}}{m_{q}}\right)$. 
In the case of the $K_{S}-K_{L}$ mass difference, the K-M angle factors make the charm quark the origin of the most important short-distance contributions and the bound that results in this case ${ }^{|6|}$ is $\left(\frac{\xi}{\eta}\right)^{2}<O\left(\frac{M_{H}}{m_{e}}\right)$.

If we turn instead to the imaginary, $\mathrm{CP}$ violating, part of the mass matrix for the neutral $K$ system, then the top quark plays a leading role. The resulting bounds that follow ${ }^{[7]}$ from making a similar assumption on the magnitude of Higgs exchange contributions versus those due to $W$ exchange are of the form $\left(\frac{\xi}{\eta}\right)^{2}<O\left(\frac{M_{H}}{m_{t}}\right)$. Since $\frac{m_{t}}{m_{c}}$ appears experimentally ${ }^{[12]}$ to be about 30 , these bounds on $\left(\frac{\xi}{\eta}\right)^{2}$ are "better" by approximately this factor. However, there is nothing sacred in making the assumption that the Higgs contributions are less than those due to $W$ 's. If we were to drop this assumption, and instead just demand consistency with the observed real and imaginary parts of the neutral $K$ mass matrix, then the above bounds are no longer in force, and we are able to use the freedom in values of the K-M angles (particularly $\sin \delta$ ) to obtain a fairly wide range of Higgs masses and values of $\frac{\xi}{\eta}$.

We can avoid the necessity of making such an assumption by going to the neutral $B$ meson system. Here the $t$ quark contribution is completely dominant in the expression for the mass difference, since it is weighted by K-M angle factors whose magnitude is like those for the charm quark, but $m_{t}^{2} \gg m_{c}^{2}$. Furthermore, the freedom in choosing matrix elements and in K-M angle related factors is considerably smaller (there is negligible dependence on $\sin \delta$ ) than in the $\mathrm{K} \mathrm{me}$ son system. Thus we can expect a bound of the form $\left(\frac{\xi}{\eta}\right)^{2}<O\left(\frac{M_{k x}}{m_{t}}\right)$ without additional assumptions on the relative magnitude of the Higgs and $W$ exchange contributions. 
Now we proceed to analyze the $B^{0}-\bar{B}^{0}$ system in detail. The off-diagonal element of the mass matrix between states whose quark content is $b \bar{d}$ and $d \bar{b}$ has both a dispersive and an absorptive part. It was already known ${ }^{[13 \mid}$ that $\left|\Gamma_{12} / M_{12}\right|=O\left(\frac{m_{b}^{2}}{m_{t}^{2}}\right) \ll 1$ for the box diagram contribution involving $W$ 's. We have checked that this also true for the Higgs contribution. Therefore $\left|\Gamma_{12}\right| \ll$ $\left|M_{12}\right|$ and $\Delta M=M_{B_{L}}-M_{B_{S}}=2\left|M_{12}\right|$. The short distance contributions to $M_{12}$ are easy to transcribe from those for the $\mathrm{K}$ system: ${ }^{[0,13]}$

$$
\begin{aligned}
M_{12}^{W W} & =\frac{G_{F}^{2} f_{B}^{2} m_{B} B_{B}}{12 \pi^{2}}\left(U_{t b}^{*} U_{t d}\right)^{2} m_{t}^{2} \\
M_{12}^{W H} & =\frac{G_{F}^{2} f_{B}^{2} m_{B} B_{B}}{3}\left(U_{t b}^{*} U_{t d}\right)^{2}\left(\frac{\xi}{\eta}\right)^{2}\left(8 M_{W}^{2} I_{2}+2 I_{3}\right) m_{t}^{4} \\
M_{12}^{H H} & =\frac{G_{F}^{2} f_{B}^{2} m_{B} B_{B}}{3}\left(U_{t b}^{*} U_{t d}\right)^{2}\left(\frac{\xi}{\eta}\right)^{4} I_{1} \frac{m_{t}^{4}}{M_{W}^{2}} .
\end{aligned}
$$

Here matrix elements of the effective Hamiltonian have been taken, neglecting ${ }^{[14]}$ terms involving external quark masses and momenta as small compared to the dominant term involving $m_{t}^{2}$ or $m_{t}^{4}$, which alone has been retained.

We have reverted to the usual practice of expressing the matrix element as a factor $B_{B}$ times its value in the vacuum insertion approximation, $\frac{4}{3} f_{B}^{2} m_{B}$, where $f_{B}$ is defined analogously to the pion or kaon decay constants, $f_{\pi}$ and $f_{K}$, and $m_{B}$ is the mass of the $B$ meson. The quantities $I_{1}, I_{2}$, and $I_{3}$ depend on $m_{t}$ and $M_{H}$ and arise from the loop integration; they are given explicitly in the appendix of Ref. 6. The $U_{i j}$ are elements of the Kobayashi-Maskawamatrix. ${ }^{[10 \mid}$ In the excellent approximation of setting the cosines of the angles $\theta_{1}, \theta_{2}$ and $\theta_{3}$ equal to unity, the elements of relevance here are $U_{t b} \approx-e^{+i \delta}$ and $U_{t d}=\sin \theta_{1} \sin \theta_{2}$. 
The connection to experiment is made through the observation that a nonzero value of $M_{12}$ (or $\Gamma_{12}$ ) will result in mixing as the weak eigenstates $B_{L}$ and $B_{S}$ with masses $M_{L}, M_{S}$ and widths $\Gamma_{L}, \Gamma_{S}$ will be mixtures of the $B^{0}$ and the $\bar{B}^{0}$. If we use the sign of the lepton charge in the semileptonic decay as an indicator of whether the decaying meson contains $a b$ or $\bar{b}$ quark, then a quantitative measure of the mixing ${ }^{(25)}$ is given by the time integrated probability for decay into a "wrong" sign lepton compared to decay into a "right" sign lepton: ${ }^{[18]}$

$$
r_{0}=\frac{\Gamma\left(B^{0} \rightarrow l^{-}+\cdots\right)}{\Gamma\left(B^{0} \rightarrow l^{+}+\cdots\right)}, \quad \bar{r}_{0}=\frac{\Gamma\left(\bar{B}^{0} \rightarrow l^{+}+\cdots\right)}{\Gamma\left(\bar{B}^{0} \rightarrow l^{-}+\cdots\right)} .
$$

Neglecting the effects of possible CP violation, which should be a good approximation in this case, ${ }^{[13]} r_{0}=\bar{r}_{0}$ and we have the expression

$$
r_{0}=\frac{(\Delta M)^{2}+(\Delta \Gamma / 2)^{2}}{2 \Gamma_{a v}^{2}+(\Delta M)^{2}-(\Delta \Gamma / 2)^{2}}
$$

where $\Delta M=M_{S}-M_{L}, \Delta \Gamma=\Gamma_{S}-\Gamma_{L}$ and $\Gamma_{a v}=\left(\Gamma_{L}+\Gamma_{S}\right) / 2$. As noted previously, $\left|\Gamma_{12}\right| \ll\left|M_{12}\right|$ and so we can neglect $\Delta \Gamma$ compared to $\Delta M$ and obtain the result relevant to the case at hand,

$$
r_{0}=\frac{(\Delta M / \Gamma)^{2}}{2+(\Delta M / \Gamma)^{2}}
$$

In present experiments one does not tag individual initial $B^{0}$ or $\bar{B}^{0}$ mesons and follow their subsequent semileptonic decay. Instead one looks at production of a pair of hadrons containing initially $a b$ and $a \bar{b}$ quark and measures the net number of same-sign and opposite-sign dileptons that result when both the heavy hadrons undergo semileptonic decay. In a situation where there is an 
uncorrelated pair of $B^{0}$ and $\bar{B}^{0}$ mesons, the ratio of same-sign to opposite-sign dileptons is ${ }^{|23,18,17|}$

$$
r=\frac{N\left(l^{+} l^{+}\right)+N\left(l^{-} l^{-}\right)}{N\left(l+l^{-}\right)+N\left(l-l^{+}\right)}=\frac{2 r_{0}}{1+r_{0}^{2}}
$$

Such would be the case generally at PEP and PETRA. However, when observing the same ratio near threshold where the $B^{\circ}$ and $\bar{B}^{\circ}$ are pair produced without other particles, the interference of the decay amplitudes (which are then coherent) results in ${ }^{[15,17]}$

$$
r=r_{0}
$$

This is the situation at CESR where an upper limit on the mixing corresponding to ${ }^{|18|}$

$$
r<0.30
$$

for the $B_{d}^{0}-\bar{B}_{d}^{0}$ system has been obtained. Applying Eqs. (2.7)and (2.5), this translates to the bound

$$
|\Delta M / \Gamma|<.93
$$

With a $B$ lifetime of $\mathbf{1 . 0}$ picosecond, we may alternately express this result as $|\Delta M|<6.1 \times 10^{-13} \mathrm{GeV}$. Note that because the limit is obtained experimentally below the $B_{s}^{0}=\bar{b}_{s}$ threshold we need not worry about another origin ${ }^{[10,20]}$ for the mixing other than that involving $B_{d}^{0}=\bar{b} d$.

Since calculations of $\mathrm{r}$ in the standard model without extra Higgs contributions typically yield predictions ${ }^{[21]}$ in the 0.01 to 0.1 range, it is clear already 
at this point that the short-distance Higgs contribution cannot be many times larger than that due to the usual $W$ contribution, or we will be in violation of the experimental bound in Eq. (2.11). From Eqs. (2.1) and (2.3) we see that

$$
\frac{M_{12}^{H} H}{M_{12}^{W W}}=4 \pi^{2}\left(\frac{\xi}{\eta}\right)^{4} m_{t}^{2} I_{1} \approx \frac{1}{4}\left(\frac{\xi}{\eta}\right)^{4} \frac{m_{t}^{2}}{M_{H}^{2}}
$$

where we have inserted ${ }^{[6]} I_{1}=\left(16 \pi^{2} M_{H}^{2}\right)^{-1}$, which is good to order $m_{t}^{2} / M_{H}^{2}$. Thus we can see that we are headed for bounds of the general form $(\xi / \eta)^{2}<$ several $\times\left(M_{H} / m_{t}\right)$.

Let us now make this more quantitative. For the moment we neglect $M_{12}^{H W}$ and use the approximate expression for $I_{1}$ given above. Then noting that $M_{12}^{W W}$ and $M_{12}^{H}{ }^{H}$ have the same phase, we have that

$$
\Delta M=2\left|M_{12}^{W W}+M_{12}^{H H}\right|=2\left|M_{12}^{W W}\right|+2\left|M_{12}^{H} H\right|
$$

and using Eqs. (2.1) and (2.3) this becomes:

$$
\Delta M=\frac{G_{F}^{2} f_{B}^{2} m_{B} B_{B} s_{1}^{2} s_{2}^{2} m_{t}^{2}}{6 \pi^{2}}\left(1+\frac{1}{4}\left(\frac{\xi}{\eta}\right)^{4} \frac{m_{t}^{2}}{M_{H}^{2}}\right)
$$

With a "nominal" set of values (discussed below) of $m_{t}=45 \mathrm{GeV}, m_{B}=5.3$ $\mathrm{GeV}, f_{B}=f_{K}=0.16 \mathrm{GeV}, s_{2}=0.06, B_{B}=1$, and a $B$ lifetime ${ }^{[22]}$ of 1.0 picosecond, this becomes the bound (shown in Fig. 2, dashed line)

$$
\left(\frac{\xi}{\eta}\right)^{2}<4.1\left(\frac{M_{H}}{m_{t}}\right)
$$

when combined with Eq. (2.12) coming from the experimental bound on the mixing. 
We now consider the bound obtained by including $M_{12}^{W H}$ and keeping the full expressions for the quantities $I_{1}, I_{2}$, and $I_{3}$ in the equation

$$
\frac{\Delta M}{\Gamma}=\frac{2\left|M_{12}^{W W}+M_{12}^{W H}+M_{12}^{H}\right|}{\Gamma}<.93
$$

from combining $\Delta M=2\left|M_{12}\right|$ with the experimental limit in Eq. (2.12). The bound that results from Eq. (2.14) is shown as the solid line in Fig. 2 using the same set of "nominal" values of the parameters as before. The approximate result of Eq. (2.15) is quite close to this exact bound, showing that it is $M_{12}^{H}{ }^{H}$ rather than $M_{12}^{H W}$ that is driving the bound. It should be noted at this point that although we have plotted the bound derived from the full expression in Eq. (2.14) as a function of $\frac{M_{B H}}{m_{t}}$ to facilitate comparison with previous bounds (e.g. Eq. (2.16) and Ref. 7), the analytic expression depends on $M_{H}$ and $m_{t}$ separately and not just on their ratio. We have set $m_{t}=45 \mathrm{GeV} / \mathrm{c}^{2}$ in plotting Fig. 2, leaving $M_{H}$ as the variable quantity.

A comment is in order here on the set of "nominal" values of the parameters which we have chosen, and their possible variation. The mass of the $B$ meson is accurately fixed by experiment and we have taken $m_{t}=45 \mathrm{GeV} / \mathrm{c}^{2}$. We equate the $B^{0}$ meson lifetime with that determined for a mixture of hadrons containing the $b$ quark, and take ${ }^{[22]} 1.0$ picoseconds for this " $b$ quark lifetime." In fact, $\tau_{b}$ enters both the value for $\sin \theta_{2}$ (from the method of determining the K-M angles) and $\Gamma_{B_{0}}$ in such a way as to cancel out in $\frac{\Delta M}{\Gamma}$, the quantity of relevance here to the mixing. So, if we use a given lifetime consistently there is no actual dependence on $\tau_{b}$. 
The value of $\sin \theta_{2}$ is extracted from $\tau_{b}$, which yields ${ }^{[23]}\left|\sin \theta_{3}+\sin \theta_{2} e^{i \delta}\right| \approx$ $0.06\left(10^{-12} \mathrm{sec} / \tau_{b}\right)^{\frac{1}{2}}$, and from the upper limit ${ }^{[24]}$ on $(b \rightarrow u) /(b \rightarrow c)$, which limits $\sin \theta_{3} /\left|\sin \theta_{3}+\sin \theta_{2} e^{i \delta}\right|<0.7$. This still allows considerable latitude in values of $\sin \theta_{2}$, from roughly $0.02\left(10^{-12} / \tau_{b}\right)^{\frac{1}{2}}$ to $0.10\left(10^{-12} / \tau_{b}\right)^{\frac{1}{2}}$.

The quantities $f_{B}$ and $B_{B}$ enter together in the form $\frac{4}{3} B_{B} f_{B}^{2} m_{B}$ as the value of the matrix element of the effective operator relevant to the short-distance contribution to $B^{\circ}-\bar{B}^{\circ}$ mixing. Several calculations of $f_{B}$ indicate ${ }^{[25 \mid}$ that $f_{B} \approx$ $f_{K} \approx f_{\pi}$, although substantially larger values ${ }^{[26]}$ have also been used. One can separately argue ${ }^{[23 \mid}$ that $B_{B} \approx 1$. Alternatively one can look at the value of the whole matrix element. Recent estimates ${ }^{[27]}$ can be rephrased as $B_{B} \approx \frac{1}{3}$ if we fix $f_{B}=f_{K}=160 \mathrm{MeV}$.

Consequently we show in Fig. 3 what happens to the bound under reasonable pessimistic $\left(B_{B}=\frac{1}{3}, \sin \theta_{2}=0.04\right.$, other parameters fixed) and optimistic $\left(B_{B}=\right.$ $\frac{3}{2}, \sin \theta_{2}=0.08$, other parameters fixed) excursions of the parameters. Even in the "pessimistic case," the bound is quite restrictive $\left(\left(\frac{\xi}{\eta}\right)^{2} \lesssim 12 M_{H} / m_{t}\right)$. From the recently obtained result in the Argus experiment, ${ }^{[28]} 20 \%$ mixing for $B_{0}-\bar{B}_{0}$ we get $(\Delta M / \Gamma)_{B} \simeq 0.7$. This corresponds to $(\xi / \eta)^{2} \simeq 10\left(M_{H} / m_{t}\right)$ in the pessimistic case and $(\xi / \eta)^{2} \simeq 1.4\left(M_{H} / m_{t}\right)$ in the optimistic one, if we use Eq.(2.15).

These limits are not far from what was obtained in Ref. 7 using the magnitude of $C P$ violation in the neutral $K$ system, but with the additional assumption in the $K$ system that the Higgs contribution be less than that of the $W$ to $\epsilon$. This is seen in Fig. 4 where this previous bound is shown as the dotdashed line, and the new bound from the $B$ system is shown as the solid line. In both cases we knew in advance that the $t$ quark short-distance contribution is dominant over 
that of the $c$ quark and consequently the bound will be of the qualitative form $\left(\frac{\xi}{\eta}\right)^{2}<O\left(M_{H} / m_{t}\right)$. The only question was the detailed number that replaces the order of magnitude: we have found that present limits of the $B^{\circ}-\bar{B}^{0}$ mixing are already able to make the new bound comparable to the previous one.

Looked at the other way, from the viewpoint of the neutral $K$ system, we see that the Higgs short-distance contribution to $\epsilon$ is not many times bigger than the standard short-distance contribution (involving $W$ 's). While the most extreme scenarios contemplated in Ref. 7 are thus ruled out, it is still quite acceptable with present limits on $B^{0}-\bar{B}^{0}$ mixing to have a major part of $\epsilon$ come from the short-distance contribution involving charged Higgs bosons. In such a situation, as emphasized in Ref. 7 , the ratio $\epsilon^{\prime} / \epsilon$ is correspondingly reduced from the value it would have in the standard model without additional Higgs. Therefore small predicted values of $\epsilon^{\prime} / \epsilon$ are still possible through the introduction of a second Higgs doublet, even with the bound on the couplings derived here from the $B^{0}-\bar{B}^{0}$ system.

\section{Limits from Toponium Spectroscopy}

We now move from a discussion of the effects of the charged Higgs to those of the neutral Higgs (with enhanced couplings), particularly on $t \bar{t}$ spectroscopy. Of all $q \bar{q}$ systems, $t \bar{t}$ is the best system to observe the neutral Higgs effects since the Higgs coupling to quarks is proportional to $m_{q}$, and relativistic effects are negligible. We begin with a review of heavy quarkonium systems. These systems are well described by treating the quarks as non-relativistic fermions interacting 
through a simple phenomenological potential, specified by a few parameters determined by fitting to the measured spectra. For the $c$ and $b$ quark systems, $a$ wide range of successful forms have been proposed. ${ }^{[20]} \mathrm{A}$ few examples are:

1. Martin: ${ }^{[30 \mid}$

$$
V(r)=(5.82 \mathrm{GeV})\left(\frac{\mathrm{r}}{1(\mathrm{GeV})^{-1}}\right)^{.104}
$$

2. Cornell: ${ }^{[32]}$

$$
V(r)=\frac{-.48}{r}+\frac{r}{\left(2.34(G e V)^{-1}\right)^{2}}
$$

3. Richardson: ${ }^{[32]}$

$$
V(r)=\frac{8 \pi}{33-2 n_{f}} \Lambda\left(\Delta r-\frac{f(\Delta r)}{\Delta r}\right)
$$

where

$$
f(t)=\left[1-4 \int_{1}^{\infty} \frac{d q}{q} \frac{e^{-q t}}{\left[\ln \left(q^{2}-1\right)\right]^{2}+\pi^{2}}\right],
$$

and $n_{f}$ is the number of quarks with mass less than the momentum of the bound heavy quarks (the relevant momentum scale for renormalization), and is taken to be 3 .

The first potential is motivated purely by the $c \bar{c}$ and $b \bar{b}$ data, while the other two incorporate to some extent the short and long range behavior expected on theoretical grounds.

The consistency of present data with potentials having widely differing analytic forms is not as surprising as it might at first seem. If one adds an appropriate constant to each potential, one finds all potentials to be in very good agreement 
in the range $.1 \mathrm{fm}<r<1 \mathrm{fm}$-where the RMS radii of the observed charmonium and bottomonium states lie (see Fig. 2 of Ref. 29 ). Toponium, however, will discriminate between these potentials-its lowest lying state may have a radius of $.05 \mathrm{fm}$ or less, depending on the potential, and the predicted level spectra for top vary widely (see Table 1 -note that the radii are specified in $\mathrm{GeV}^{-1}$ ).

Into this somewhat murky situation of differing strong interaction potentials we now introduce the added effects of neutral Higgs boson exchange (Fig. 5). The analogue of Eq. (2.1) for charged Higgs is ${ }^{\text {[s] }}$

$$
\begin{aligned}
\mathcal{L}_{\text {int }} & =\frac{g}{2 M_{W}} \phi_{1}\left\{\bar{U}\left[\frac{\left(\xi^{2}+\eta^{2}\right)^{1 / 2}}{\eta} M_{u}\right] U \cos \beta+\bar{D}\left[\frac{\left(\xi^{2}+\eta^{2}\right)^{1 / 2}}{\xi} M_{d}\right] D \sin \beta\right\} \\
& +\frac{g}{2 M_{W}} \phi_{2}\left\{-\bar{U}\left[\frac{\left(\xi^{2}+\eta^{2}\right)^{1 / 2}}{\eta} M_{u}\right] U \sin \beta+\bar{D}\left[\frac{\left(\xi^{2}+\eta^{2}\right)^{1 / 2}}{\xi} M_{d}\right] D \cos \beta\right\} \\
& +\frac{g}{2 M_{W}} \phi_{3}\left\{\bar{U}\left[\frac{\xi}{\eta} M_{u} \gamma_{5}\right] U+\bar{D}\left[\frac{\eta}{\xi} M_{d} \gamma_{5}\right] D\right\},
\end{aligned}
$$

where $\beta$ is an unknown mixing angle between the two scalar physical fields, $\phi_{1}^{0}$ and $\phi_{2}^{\circ}$. We will concentrate in what follows on the effects of exchange of the single pseudoscalar field $\phi_{3}^{0}$, with couplings to $t$ quarks enhanced over the usual case by the same ratio of vacuum expectation values, $\xi / \eta$, which we have already bounded. These are independent of any additional mixing angle and form a lower bound on these effects; in any case, once we know the effects of single Higgs exchange the sum of several exchanges with differing masses and couplings can be readily calculated.

In momentum space, the diagram in Fig. 5 then corresponds to adding the 
following term to the spin independent part of the non-relativistic potential:

$$
-\left(\frac{\xi}{\eta} \frac{g m_{t}}{2 M_{W}}\right)^{2} \frac{1}{m^{2}+q^{2}}
$$

which gives $^{[34]}$

$$
-\left(\frac{\xi}{\eta} \frac{g m_{t}}{2 M_{W}}\right)^{2} \frac{1}{4 \pi r} e^{-r M_{H}}
$$

in coordinate space. Again, this Yukawa-type attractive potential is to be added to whatever potential is chosen to represent the strong interactions for the $t \bar{t}$ system.

As has been noted before,${ }^{[8]}$ the energy levels and widths of toponium states will be noticeably shifted by the exchange of a Higgs with enhanced couplings. The qualitative features of its effects follow from it being attractive and having its strongest effect close to the origin (as it dies off exponentially with distance). It tends to pull in wave functions, decrease bound state radii, and increase wave functions at the origin, with its strongest effect being on the lowest lying states whose wave functions are already large in the neighborhood of the origin where the Higgs exchange potential lives.

Thus it is easy to understand the increased $E_{2 S}-E_{1 S}$ splitting in the presence of Higgs exchange, an effect already noted by Sher and Silverman: ${ }^{[8]}$ the 1S state, with a bigger wave function at the origin to begin with, is pulled down deeper into the potential well than is the $2 S$ state by the added Higgs term. However, an inspection of Table I reveals that comparable or larger differences in $E_{2 S}-E_{1 S}$ are obtained by changing from one strong interaction potential to another. By itself this effect does not decisively point to Higgs exchange as its unique origin. 
What happens to the $\mathrm{E}(2 \mathrm{~S})-\mathrm{E}(1 \mathrm{P})$ separation is not quite as obvious. The situation is elucidated by a theorem of Martin: ${ }^{(35)}$ if $\Delta V(r)=\frac{d}{d r} r^{2} \frac{d V}{d r}>0$ (true for all proposed quarkonia potentials), the nS state lies above the (n-1)P state, while if $\Delta V(r)<0$ for all $r$ such that $d V / d r>0$ (true for the Higgs potential), the $\mathrm{nS}$ state lies below the corresponding $\mathrm{P}$ state. Here we have a qualitative signature of the presence of the Higgs. However, the theorem requires the given condition on $\Delta V(r)$ to hold for all r. (The condition $d V / d r>0$ holds for both the Higgs and quarkonium potentials.) What happens in our case, where the Higgs only dominates near the origin? We might guess that the energy levels will be inverted if the Higgs term dominates below some relevant radius, perhaps that of the $2 \mathrm{~S}$ or $1 \mathrm{P}$. As $M_{H}$ increases, the range of the Higgs potential decreases and we need a larger value of $\frac{\xi}{\eta}$ to keep $\Delta V<0$. This does give a qualitative picture of what happens. To determine quantitatively the minimum value of $\frac{\xi}{\eta}$ for the level inversion, we numerically solve the Schrödinger equation. After obtaining $\mathrm{E}(2 S)$ and $\mathrm{E}(1 P)$ for various values of $\frac{\xi}{\eta}$, we interpolate to estimate the value of $\frac{\xi}{\eta}$ at which $\mathrm{E}(2 S)=\mathrm{E}(1 P)$, which is shown in Fig. 6 for both the Richardson and Cornell potentials. The Cornell potential, which starts with a bigger wave function at the origin, requires a smaller Higgs coupling enhancement to affect the inversion. We find that for large $M_{H}^{0}$ the $2 \mathrm{~S}$ level is depressed by Higgs-induced effects while the $1 \mathrm{P}$ remains much the same. As we decrease $M_{H}$ the $2 \mathrm{~S}$ becomes more and more depressed until for very small $M_{H}$ the Compton wavelength of the neutral Higgs becomes comparable to the size of the $t \bar{t}$ system and the $1 \mathrm{P}$ starts to sink almost as fast as the $2 \mathrm{~S}$; hence the rise in the curves as we go to very small $M_{H}$. 
Fairly spectacular effects can be produced in the wave function at the origin, particularly that of the lowest lying S-states. Here the part of the potential which is singular at the origin, i.e., which behaves as $\frac{1}{r}$, would be expected to play the main role. That this is indeed the case is shown in Fig. 7 where the dependence of $|\psi(0)|$ on $\frac{\xi}{\eta}$, for the 1S ground state of the $t \bar{t}$ system is plotted: there is only a very small difference between the results obtained from the full Cornell potential (solid line) and those obtained from its Coulomb-like part alone (dashed line)note the suppressed zero. Similar results are found for the Richardson potential.

This suggests separating the portion of both the strong interaction and Higgs exchange potentials which are singular as $r \rightarrow 0$ and using this combination to determine (approximately) $\psi(0)$. This effective Coulomb potential $-\frac{\tilde{\alpha}}{r}$ will have strength

$$
\widetilde{\alpha}=\frac{4}{3} \alpha_{s}+\frac{1}{4 \pi}\left(\frac{g m_{t}}{2 M_{W}}\right)^{2}\left(\frac{\xi}{\eta}\right)^{2}
$$

Since for the corresponding ground state, $|\psi(0)|^{2} \propto\left(\tilde{\alpha} m_{t}\right)^{3}$, we might expect that

$$
|\psi(0)|^{2 / 3}=|\psi(0)|_{\frac{\xi}{\eta}=0}^{2 / 3}\left[1+c(\xi / \eta)^{2}\right]
$$

where

$$
c=\frac{3}{16 \pi \alpha_{s}}\left(\frac{g m_{t}}{2 M_{W}}\right)^{2}
$$

In Fig. 7 we see that the linear behavior expected on the basis of Eq. (3.9) is a fairly good representation of the actual dependence. However, the deduced coefficient of $(\xi / \eta)^{2}$ is smaller than that predicted by Eq. (3.10), presumably because the characteristic factor of $e^{-M_{H} r}$ "screens" the full strength of the 
effective Coulomb piece of the Higgs exchange potential as we move out any finite distance from the point at $r=0$. Be that as it may, thinking of the situation in terms of a single effective Coulomb potential leads to the qualitative or even semiquantitative understanding of the behavior of $\psi(0)$ shown in Fig. 7. For light neutral Higgs $\left(M_{H^{0}} \approx 5\right.$ to $\left.20 \mathrm{GeV} / \mathrm{c}^{2}\right)$ in particular, $\psi(0)$ changes appreciably, even for moderate values of $\xi / \eta$ in the case of the Richardson potential (see Table 1).

Fig. 8 shows the effect on $|\psi(0)|$ of Higgs boson exchange with large $\frac{\xi}{\eta}$ through Z-toponium mixing ${ }^{[30]}$ (which depends on $\left|\psi_{n}(0)\right|^{2}$ ) for entire spectrum of $\mathrm{nS}$ states (for the Richardson potential), while for comparison Figs. 9 and 10 show the spectra for the Richardson and Cornell potentials, with no Higgs. The differences are fairly striking, although the Cornell potential without Higgs (which has a larger coefficient of $\frac{1}{r}$ ) partly mimics the effect of adding Higgs exchange to the Richardson potential.

We also show, in Fig. 11, the bump due to the $1 \mathrm{~S}$ state, smeared by beam energy spread, for various values of $|\psi(0)|_{1 S}$, taking $M_{V_{0}}$ fixed to be above the $Z$ at $98 \mathrm{GeV}$ (see Table 1 for a correspondence of these wavefunction values to $\frac{\xi}{\eta}$ and $\left.M_{H}\right)$. As discussed in Ref. 36, the bare width of the $1 \mathrm{~S}$ is swamped by the width it acquires from mixing; this in turn is less than or near the machine resolution. Consequently the net effect of a larger $|\psi(0)|$ is simply to make the resonance more noticeable.

We conclude, however, that in general it may be far from easy to obtain a useful bound on $\frac{\xi}{\eta}$ from this effect. The study of $B^{0}-\bar{B}^{0}$ mixing in the previous section already places a rather stringent bound on $\frac{\xi}{\eta}$; the changes in levels and 
wavefunctions in the remaining region of interest are mostly comparable to the differences in these quantities found from use of different potential models.

Still, a careful study, when toponium levels have been measured, might well yield information on the neutral Higgs boson. Certainly these effects must be borne in mind when the data has been taken, and one attempts to fit it to various potential models.

\section{Conclusion}

The bounds we have obtained from the $B^{0}-\bar{B}^{0}$ system on the ratio of vacuum expectation values, $\xi / \eta$, in the two Higgs doublet model, is a fairly tight one. For charged Higgs masses below $\approx 0.5 \mathrm{TeV}$ (where $\Gamma_{H} \ll M_{H}$ ), we have $\frac{\xi}{\eta} \lessgtr 10$, even with some pessimism on the parameters entering the bound. If we narrow the region of interest for $M_{H}+$ to be the more accessible one below a couple of hundred $\mathrm{GeV} / \mathrm{c}^{2}$, then $\xi / \eta \lessgtr 5$ with the nominal set of parameters we have been using.

As we have noted several times, this is comparable to the bound obtained from the neutral $\mathrm{K}$ system, but with the added assumption there that the Higgs shortdistance contribution to the CP violation parameter $\epsilon$ is less than the standard short-distance contribution involving $W$ 's. It is also comparable or better than bounds on $\xi / \eta$ coming from other sources. For example, the bound $\xi / \eta \lessgtr$ $2 M_{H^{+}} /\left(9 m_{\mathrm{c}} m_{t}\right)^{1 / 2}$, derived in Ref. 8 from an assumed agreement of the $t$-quark semileptonic branching ratio with that of the standard model, is considerably less stringent than ours when $M_{H^{+}}>m_{t}$. Recently a bound on $\xi / \eta$ which is 
independent of $M_{H^{+}}$has been derived ${ }^{[37 \mid}$ from the assumption of perturbative grand unification of $\mathrm{SU}(3) \times \mathrm{SU}(2) \times \mathrm{U}(1)$ with a desert between the weak and unification scales. For values of $M_{H+}$ below several hundred $\mathrm{GeV}$ the bound on $\xi / \eta$ obtained from the $B^{0}-\bar{B}^{0}$ system is smaller, while for larger $M_{H^{+}}$the bound of Ref. 36 is the more restrictive one. Quite tight bounds ${ }^{[38]}$ on $\xi / \eta$, also follow from the requirement of stability of the Higgs potential when the lighter neutral scalar Higgs has a low mass.

The limits on $\xi / \eta$ found from the $B^{0}-\bar{B}^{0}$ system dampen the enthusiasm one feels at first sight for the potentially dramatic effects in the $t \bar{t}$ system due to exchange of a neutral Higgs boson with enhanced couplings, e.g., enlarged $\mathrm{E}(2 \mathrm{~S})-\mathrm{E}(1 \mathrm{~S})$ splittings, enhanced $|\psi(0)|$, etc. Once we restrict ourselves to say, $\xi / \eta<5$, the effects are not enormous unless $M_{H^{0}}$ is quite small. Furthermore, exactly in cases where the effects are not large, they are qualitatively similar to the effects obtained by changing from one strong interaction potential to another with a stronger $\frac{1}{r}$ singularity. In this regard, we emphasized the inversion of the $2 S$ and $1 \mathrm{P}$ levels as something which is qualitatively different in the presence of a Higgs exchange potential of sufficient strength. But even for this property, Fig. 6 shows that values of $\xi / \eta<5$ are not sufficient to cause this level inversion for the Richardson potential and do so only for small $M_{H^{\circ}}$ in the case of the Cornell potential.

Nevertheless, a large value of $M_{H^{ \pm}}$(yielding a weaker bound on $\xi / \eta$ ) together with a small value of $M_{H^{0}}$ for at least one of the neutral Higgs bosons in the two doublet model is a possible scenario to contemplate. In such a case, by carefully comparing the $t \bar{t}$ spectrum and wave functions in several of its aspects 
simultaneously, it still could be possible to sort out the effects of neutral Higgs exchange from those of differing strong interaction potentials. 


\begin{tabular}{|c|c|c|c|c|c|c|c|c|c|}
\hline Potential & $M_{H}$ & $\frac{\xi}{n}$ & $E_{1 s}$ & $\langle r\rangle_{1 S}$ & $E_{28}-E_{18}$ & $E_{2 s}-E_{1 p}$ & $\Psi(0)_{18}$ & $\begin{array}{l}\Psi(0)_{20} \\
\Psi(0)_{10}\end{array}$ & $\Psi^{\prime}(0)_{2 p}$ \\
\hline \multirow[t]{14}{*}{ Richardson } & - & 0 & 98.323 & .24 & .999 & .102 & 8.5 & .52 & 13.0 \\
\hline & 5 & 2 & 98.277 & .235 & 1.04 & .099 & 9.0 & .506 & 13.6 \\
\hline & 5 & 7 & 97.609 & .18 & 1.58 & .061 & 15.1 & .42 & 21.9 \\
\hline & 10 & 3 & 98.259 & .231 & 1.05 & .093 & 9.3 & .50 & 13.8 \\
\hline & 10 & 6 & 98.027 & .204 & 1.24 & .063 & 12.3 & .45 & 16.4 \\
\hline & 10 & 8 & 97.708 & .177 & 1.51 & .032 & 16.2 & .40 & - \\
\hline & 10 & 11 & 96.739 & .133 & 2.37 & -.037 & 26.4 & .306 & 29.3 \\
\hline & 20 & 2 & 98.31 & .238 & 1.009 & .099 & 8.76 & .51 & 13.2 \\
\hline & 20 & 6 & 98.189 & .22 & 1.103 & .075 & 11.1 & .48 & 14.6 \\
\hline & 20 & 10 & 97.817 & .18 & 1.41 & .016 & 18.0 & .41 & 17.9 \\
\hline & 40 & 3 & 98.312 & .238 & 1.007 & .099 & 8.83 & .51 & 13.2 \\
\hline & 40 & 8.2 & 98.228 & .224 & 1.071 & .08 & 11.4 & .49 & 14.3 \\
\hline & 80 & 7 & 98.304 & .237 & 1.013 & .097 & 9.47 & .51 & 13.5 \\
\hline & 140 & 7 & 98.316 & .238 & 1.004 & .10 & 9.01 & .51 & 13.3 \\
\hline \multirow[t]{5}{*}{ Cornell } & - & 0 & 97.143 & .144 & 2.226 & .015 & 23.3 & .372 & 30.8 \\
\hline & 10 & 2 & 97.067 & .141 & 2.29 & .010 & 24.0 & .368 & 31.6 \\
\hline & 10 & 4 & 96.823 & .133 & 2.51 & -.005 & 26.4 & .354 & 34.3 \\
\hline & 40 & 4 & 97.055 & .14 & 2.30 & .005 & 24.9 & .366 & 31.6 \\
\hline & 100 & 6 & 97.09 & .141 & 2.27 & .008 & 24.9 & .369 & 31.4 \\
\hline \multirow[t]{4}{*}{ Martin } & - & 0 & 98.628 & .419 & .455 & .127 & 2.72 & .75 & 4.41 \\
\hline & 10 & 5 & 98.58 & .388 & .482 & .109 & 3.74 & .70 & 5.11 \\
\hline & 40 & 5 & 98.624 & .416 & .457 & .125 & 3.00 & .74 & 4.56 \\
\hline & 100 & 5 & 98.627 & .419 & .456 & .127 & 2.83 & .75 & 4.47 \\
\hline
\end{tabular}

Table 1. Calculated parameters of toponium, for a few different potentials, 
values of $M_{H}$, and $\frac{\xi}{\eta} ; m_{t}=50 \mathrm{GeV}$ (all units $\mathrm{GeV}$ to appropriate powers). 


\section{REFERENCES}

1. S. Weinberg, Phys. Rev. Lett. 19, 1264 (1967); A. Salam, in Elementary Particle Theory: Relativistic Groups and Analyticity (Nobel Symposium No.8), edited by N. Svartholm (Almqvist and Wiksell, Stockholm, 1968), p. 367; S. L. Glashow, J. Iliopoulos and L. Maiani, Phys. Rev. D2, 1285 (1970), referred to as GIM.

2. See for example the review in P. Langacker, Proceedings of the Summer Study on Design and Utilization of the Superconducting Supercollider, Snowmass, Colorado, June 23-July 23, 1984, edited by R. Donaldson, J. Morfin (Fermilab, Batavia, Illinois, 1985) p. 771.

3. L. Susskind, Phys. Rev. D20, 2619 (1979); E. Farhi and L. Susskind, Phys. Rept. 74, 277 (1981), and references therein.

4. J. C. Pati and A. Salam, Phys. Rev. D10, 275 (1974); G. Senjanović, Nucl. Phys. B153, 334 (1979).

5. J. Wess and B. Zumino, Nucl. Phys. B70, 39 (1974); P. Fayet, Nucl. Phys. B90, 104 (1975); S. Dimopoulos and H. Georgi, Nucl. Phys. B193, 150 (1981); N. Sakai, Z. Phys. C11, 153 (1981).

6. L. F. Abbott, P. Sikivie and M. B. Wise, Phys. Rev. D21, 1393 (1980).

7. G. G. Athanasiu and F. J. Gilman, Phys. Lett. 153B, 274 (1985).

8. M. Sher and D. Silverman, Phys. Rev. D31, 95 (1985).

9. S. Glashow and S. Weinberg, Phys. Rev. D15, 1958 (1977), E. A. Paschos, Phys. Rev. D15, 1966 (1977). 
10. M. Kobayashi and T. Maskawa, Prog. Theor. Phys. 49, 652 (1973).

11. H. E. Haber, G. L. Kane and T. Sterling, Nucl. Phys. B161, 493 (1979).

12. G. Arnison et al., Phys. Lett. 147B 493 (1984).

13. J. Hagelin, Nucl. Phys. B193 123 (1981), and references therein.

14. We have checked that the neglected terms are $O\left(m_{b}^{2} / m_{t}^{2}\right)$ or $O\left(m_{c}^{2} / m_{t}^{2}\right)$ for both the contributions involving Higgs exchange and those involving $W$ exchange. We have also not included strong interaction (QCD) corrections to the effective weak interaction operators, as they have a relatively small net phenomenological effect (see Refs. 6 and 13).

15. L. B. Okun, B. M. Pontecorvo, and V.I. Zakharov, Nuovo Cim. Lett. 13 218 (1975); A. Pais and S. B. Treiman, Phys. Rev. D12, 2744 (1975); R. L. Kingsley, Phys. Lett. 63B, 329 (1976).

16. We follow the new Particle Data Group name conventions presented by C.G.Wohl et al.,Proceedings of Santa Fe Meeting of the Division of Particles and Fields of the Amerikan Physical Society,1984,edited by T.Goldman and M.M.Nieto(World Scientific, Singapore,1985),p.192, wherein $B^{\circ}=\bar{b} d$ and $\bar{B}^{0}=b \bar{d}$.

17. I. I. Bigi and A. I. Sanda, Nucl. Phys. B193, 85 (1981); A. B. Carter and A. I. Sanda, Phys. Rev. D23, 1567 (1981).

18. P. Avery et al., Phys. Rev. Lett. 53, 1309 (1984).

19. A limit at high energy from the Mark II collaboration, T. Schaad et al., SLAC Report No.SLAC-PUB-3696, 1985 (unpublished) is consistent with 
that of Ref. 18 and results in a somewhat better limit if production of $B_{0}^{0}$ mesons is assumed together with their mixing as predicted by the standard model.

20. At high energies one has a mixture of $B_{a}^{0}, B^{+}$and $B^{0}$ mesons as well as baryons containing the $b$ quark, for lifetime as well as semileptonic decay measurements. Even near threshold dileptons can arise from both $B^{0}$ and $B^{+}$decays and therefore the present limit is dependent on the assumption that the semileptonic branching ration of the two meson is the same. See Ref. 18.

21. F. J. Gilman and J. S. Hagelin, Phys. Lett. 133B, 443 (1983); E. A. Paschos, B. Stech and U. Türke, Phys. Lett. 128B, 240 (1983); E. A. Paschos, and U. Türke, Nucl. Phys. B243, 29 (1984); S. Pakvasa, Phys. Rev. D28, 2915 (1983); T. Brown and S. Pakvasa, Phys. Rev. D31, 1661 (1985); A. Buras, W. Slominski, and H. Steger, Nucl. Phys. B245, 369 (1984); L. L. Chau, Phys. Rev. D29, 592 (1984); I. I. Bigi and A. I. Sanda, Phys. Ref. D29, 1393 (1984).

22. J. Jaros, in Proceedings of the 1984 SLAC Summer Institute, edited by P. McDonough (Stanford Linear Accelerator Center, Stanford, 1985)p.427, and references to experiments therein.

23. We use $m_{b}=4.7 \mathrm{GeV} / c^{2}$ and $m_{c}=1.5 \mathrm{GeV} / \mathrm{c}^{2}$ as in Gilman and Hagelin, Ref. 21.

24. J.Lee-Franzini, in Flavor Mixing in Weak Interactions, proceedings of the 1984 Europhysics Topical Conference,Erice,edited by L.L.Chau(Plenum,New 
York, 1984),p.217; A. Chen et al., Phys. Rev. Lett. 52, 1084 (1984).

25. H. Krasemann, Phys. Lett. 96B, 397 (1980); E. Golowich, Phys. Lett. 91B, 271 (1980); V. Novikov et al., Phys. Rev. Lett. 38, 626 (1977).

26. E. A. Paschos et al., Ref. 21; S. Pakvasa, Ref. 21.

27. I. Bigi and A. Sanda, Ref. 21.

28. ARGUS Collaboration,H.Albret et.al.,DESY preprint (to be published) as reported by H.Schroder at the XXII Rencontre de Moriont, Les Arcs,France,March 1987

29. For a review, see for example E. Eichten, in Proceedings of the 1984 SLAC Summer Institute on Particle Physics, Stanford, edited by P. McDonough (Stanford Linear Accel. Center, Stanford, 1985), (to be published), and references therein.

30. A. Martin, Phys. Lett. 93B, 338 (1980).

31. E. Eichten et al., Phys. Rev. D17, 3090 (1979); D21, 203 (1980).

32. J. L. Richardson, Phys. Lett. 82B, 272 (1979).

33. See, for example, P. Langacker, Ref. 2; M. Sher and D. Silverman, Ref. 8.

34. We write $\tilde{V}(q)$ in accordance with the convention $V(r)=\frac{1}{(2 \pi)^{3}} \int \tilde{V}(q) e^{i \vec{q} \cdot \vec{r}} d^{3} \vec{q}$. This differs from the convention used by Richardson: our $\tilde{V}(q)$ is $4 \pi$ times his.

35. A. Martin,Nucl.Phys.B254,528(1985). 
36. P. J. Franzini and F. J. Gilman,Phys.Rev.D32,237(1985); S. Güsken, J. H. Kühn, and P. M. Zerwas, SLAC-PUB3580,1980 (unpublished)J. Kühn, and P. M. Zerwas, Phys.Lett.154B,448(1985); L. J. Hall, S. F. King, and S. R. Sharpe, Nucl.Phys.B260,510(1985).

37. J. Bagger, S. Dimopoulos, and E. Massó, Phys.Lett.156B,357(1985).

38. M. Cvetið, C. Preitschopf, and M. Sher,Phys.Lett.164B,90,(1985). 


\section{FIGURE CAPTIONS}

1) Box diagrams contributing to $B^{0}-\bar{B}^{0}$ mixing in a two-Higgs doublet model. $\mathrm{H}$ is the physical, charged Higgs.

2) Limit on $\left(\frac{\xi}{\eta}\right)^{2}$ versus the charged Higgs mass from $B^{0}-\bar{B}^{0}$ mixing, for the "nominal" values of parameters given in the text. The dashed line is the approximate bound (see Eq. 2.16), while the solid curve is the full bound.

3) Possible variations due to the use of different parameters in the limit given in Fig. 2. The upper curves correspond to the "pessimistic" case described in the text; the lower to the "optimistic." The corresponding approximate bounds are denoted by dashed lines.

4) Comparison of our limit from Fig. 2 (solid curve) with those of Ref. 7 (dotdash).

5) Neutral Higgs exchange diagram contributing to the binding potential in the $t \bar{t}$ system.

6) Minimum value of $\frac{\xi}{\eta}$ for which $E_{1 P}>E_{2 S}$, versus Higgs mass, for the Richardson and Cornell potentials.

7) $|\psi(0)|^{2 / 3}$ versus $\left(\frac{\xi}{\eta}\right)^{2}$ for the Cornell potential (solid curve), and its Coulomb part alone (dashed curve), (the light dotted line is straight, for comparison). $M_{H^{0}}=40 \mathrm{GeV}$.

8) $R\left(e^{+} e^{-} \rightarrow \mu^{+} \mu^{-}\right)$resulting from toponium-Z mixing for the Richardson potential, with $m_{t}=47.5 \mathrm{GeV} / \mathrm{c}^{2}, \frac{\xi}{\eta}=12, M_{H}=10 \mathrm{GeV}$, convoluted with a gaussian appropriate for $\sigma_{\text {beam }}=40 \mathrm{MeV}$. 
9) $R\left(e^{+} e^{-} \rightarrow \mu^{+} \mu^{-}\right)$resulting from toponium-Z mixing for the Richardson potential, with $m_{t}=47.5 \mathrm{GeV} / \mathrm{c}^{2}$, but no Higgs exchange, convoluted with a gaussian appropriate for $\sigma_{\text {beam }}=40 \mathrm{MeV}$.

10) $R\left(e^{+} e^{-} \rightarrow \mu^{+} \mu^{-}\right)$resulting from toponium-Z mixing for the Cornell potential, $m_{t}=47.5 \mathrm{GeV} / \mathrm{c}^{2}$, but no Higgs exchange, convoluted with a gaussian appropriate for $\sigma_{\text {beam }}=40 \mathrm{MeV}$.

11) $R\left(e^{+} e^{-} \rightarrow \mu^{+} \mu^{-}\right)$resulting from the $1 \mathrm{~S}$ resonance, smeared by $\sigma_{\text {beam }}=40$ $\mathrm{MeV}$, for various values of $|\psi(0)|_{1 s}$, and a fixed $M_{V_{0}}=98 \mathrm{GeV}$. 

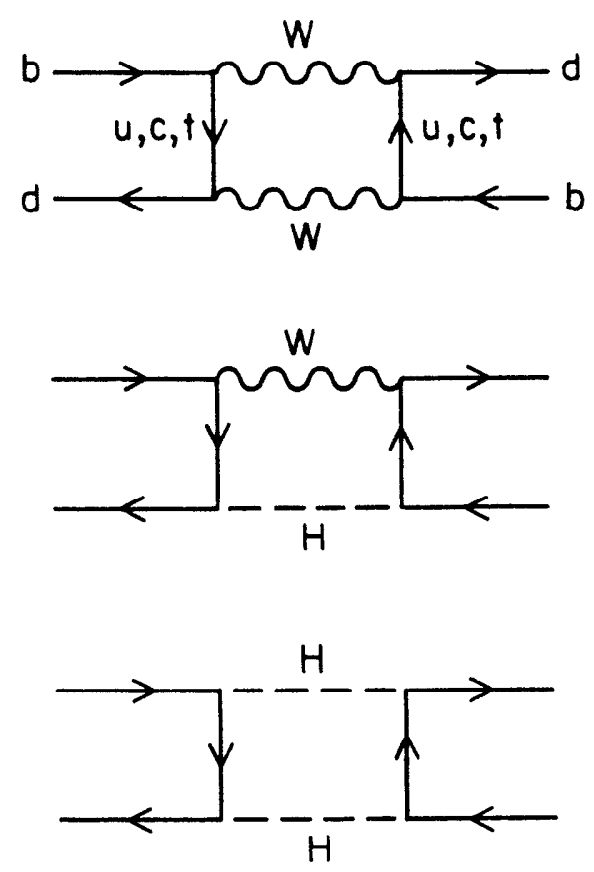
5-85
$5147 \mathrm{~A} 1$

FIGURE 1 


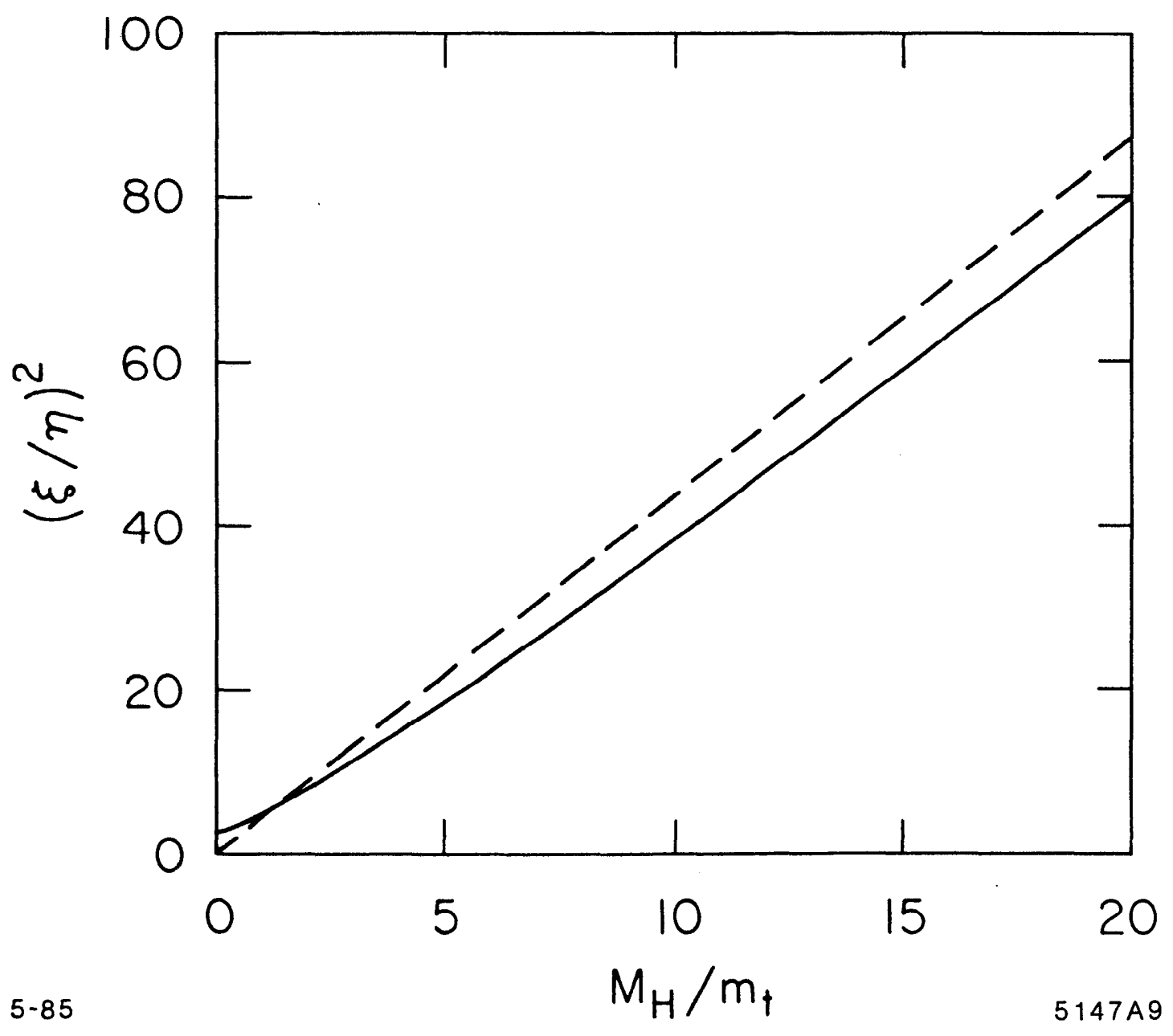

FIGURE 2 


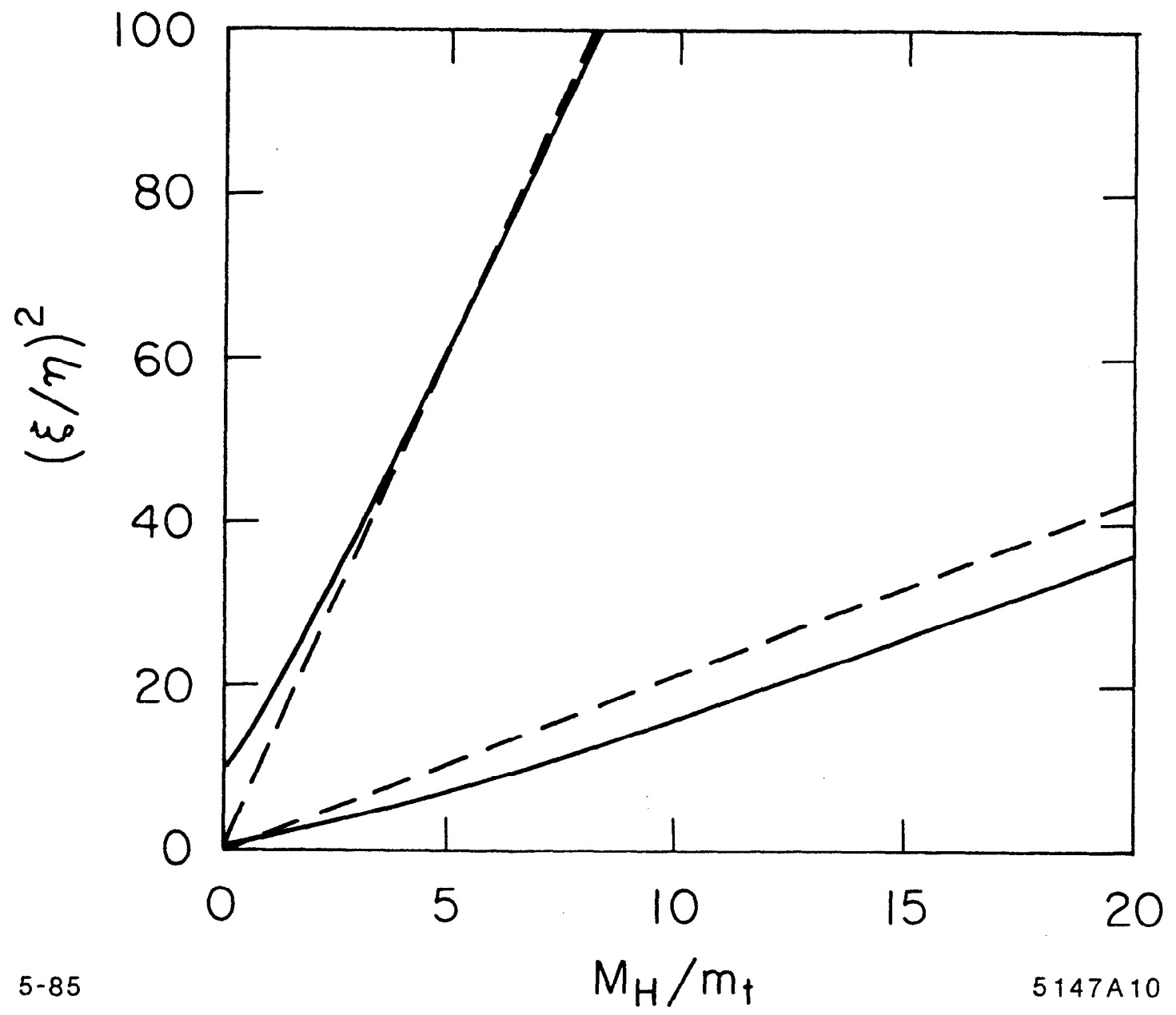

FIGURE 3 


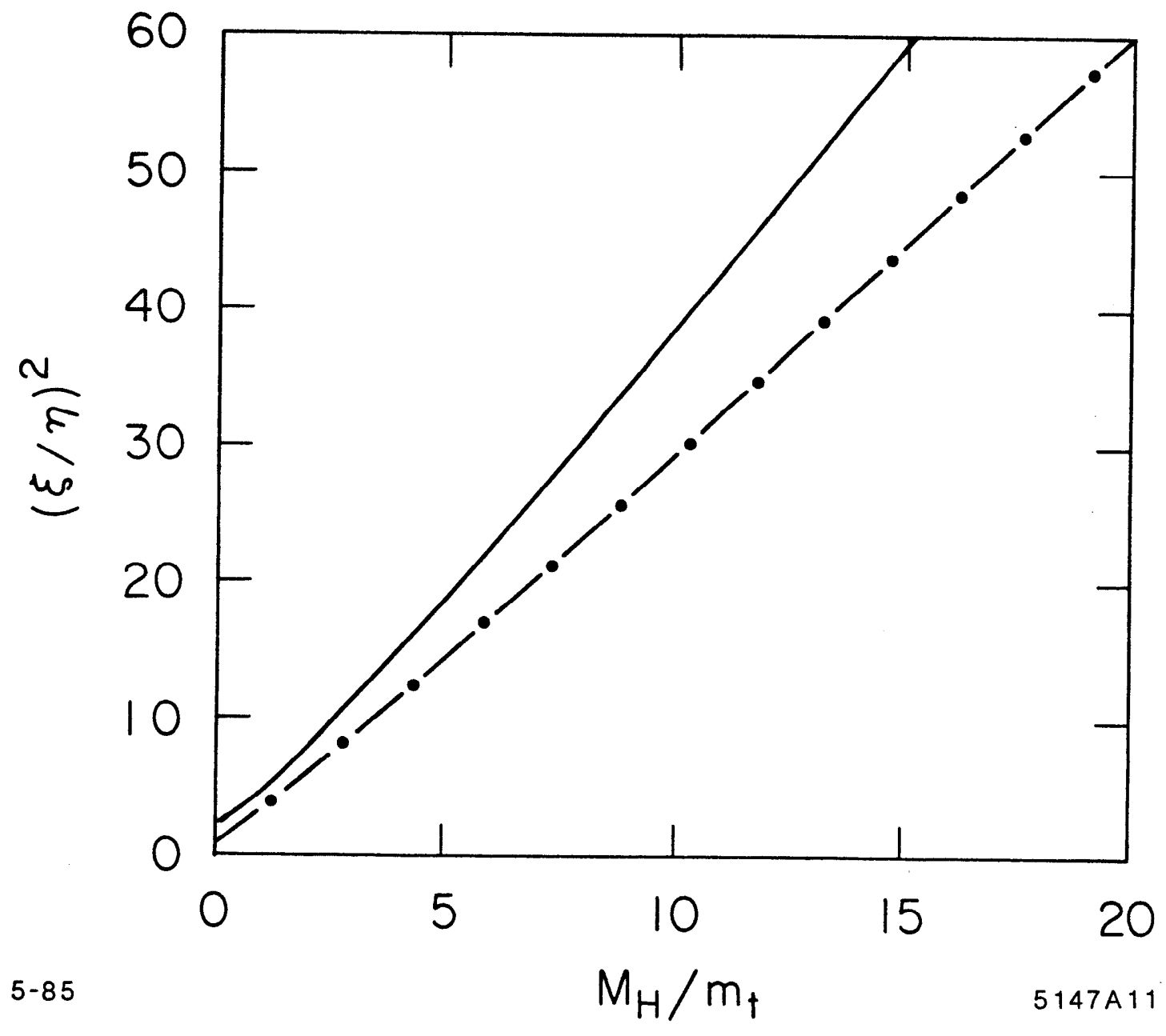

FIGURE 4 


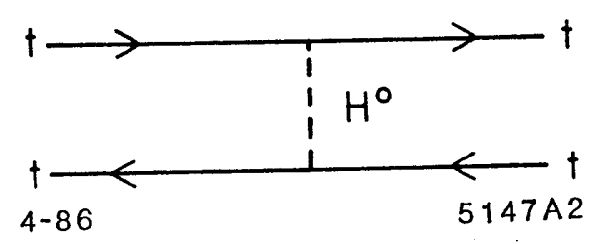

FIGURE 5 


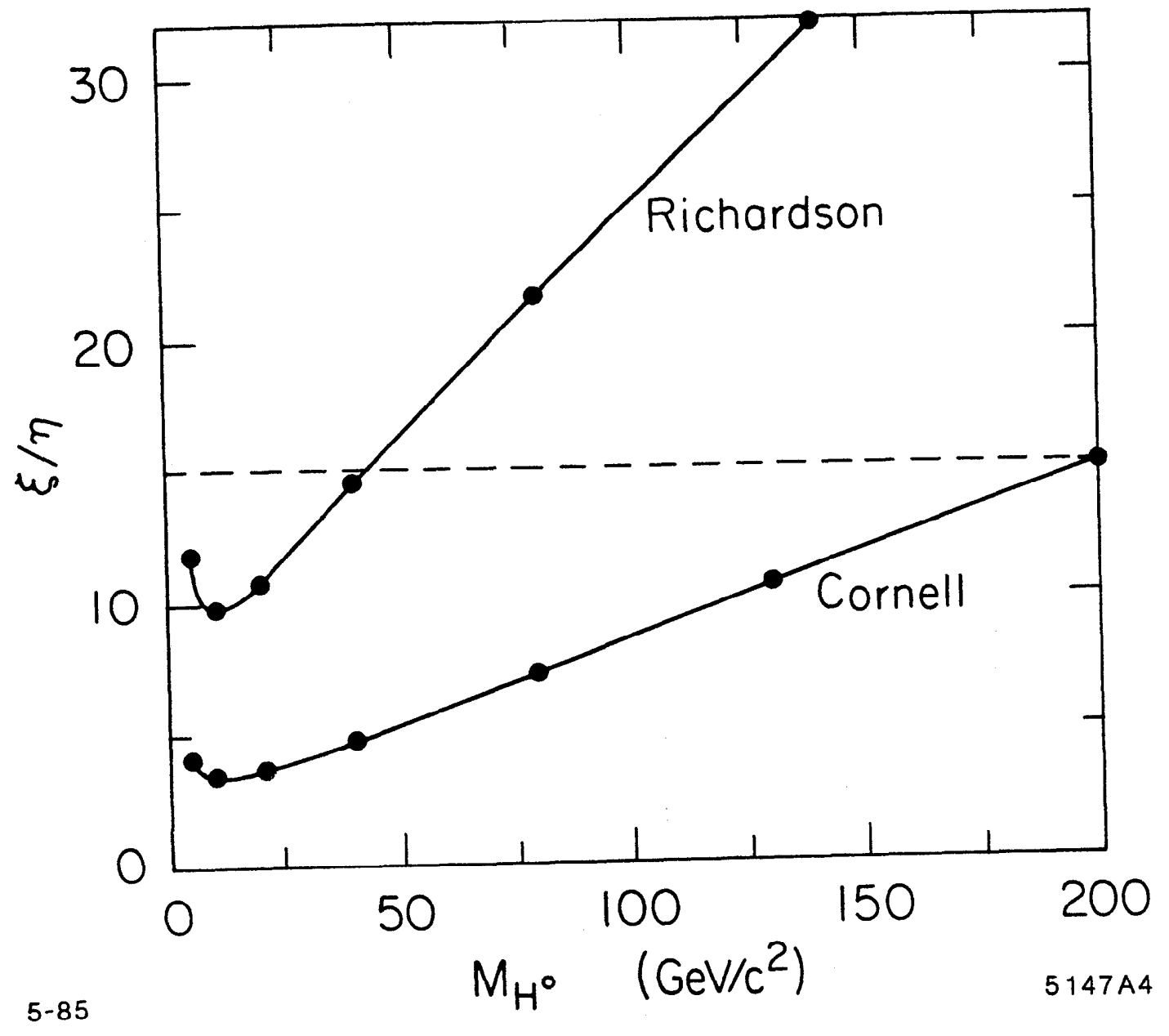

FIGURE 6 


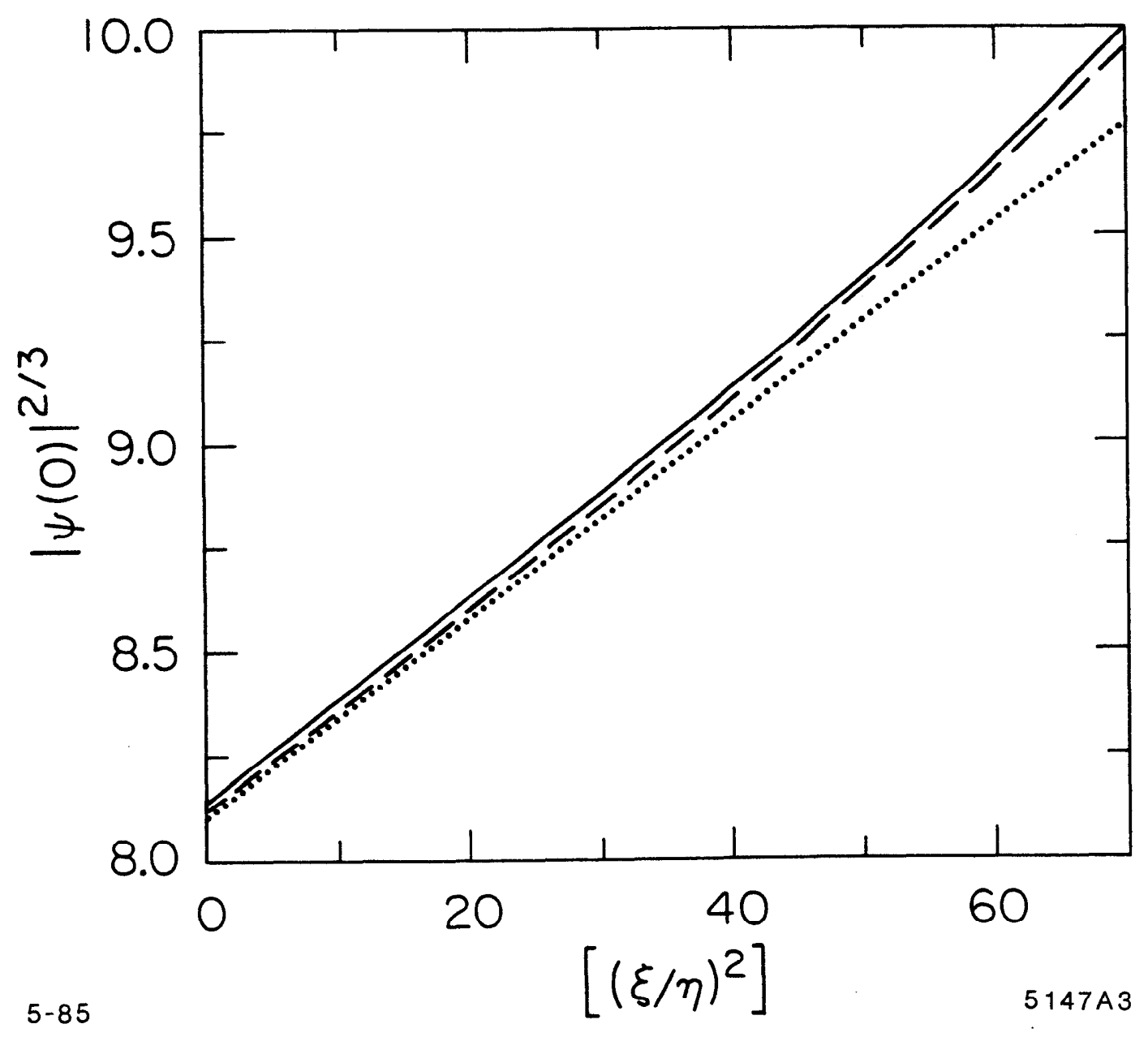

FIGURE 7 


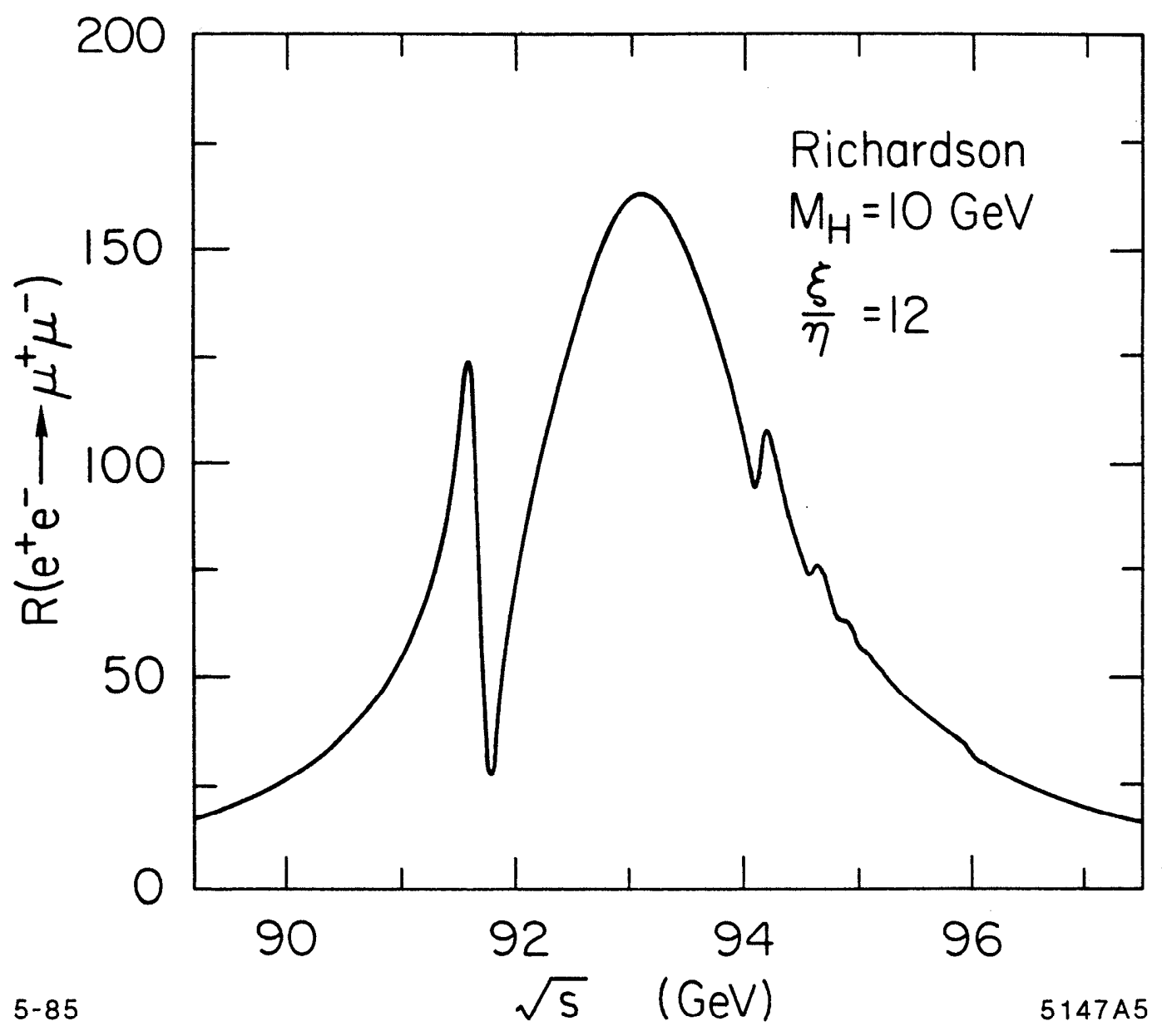

FIGURE 8 


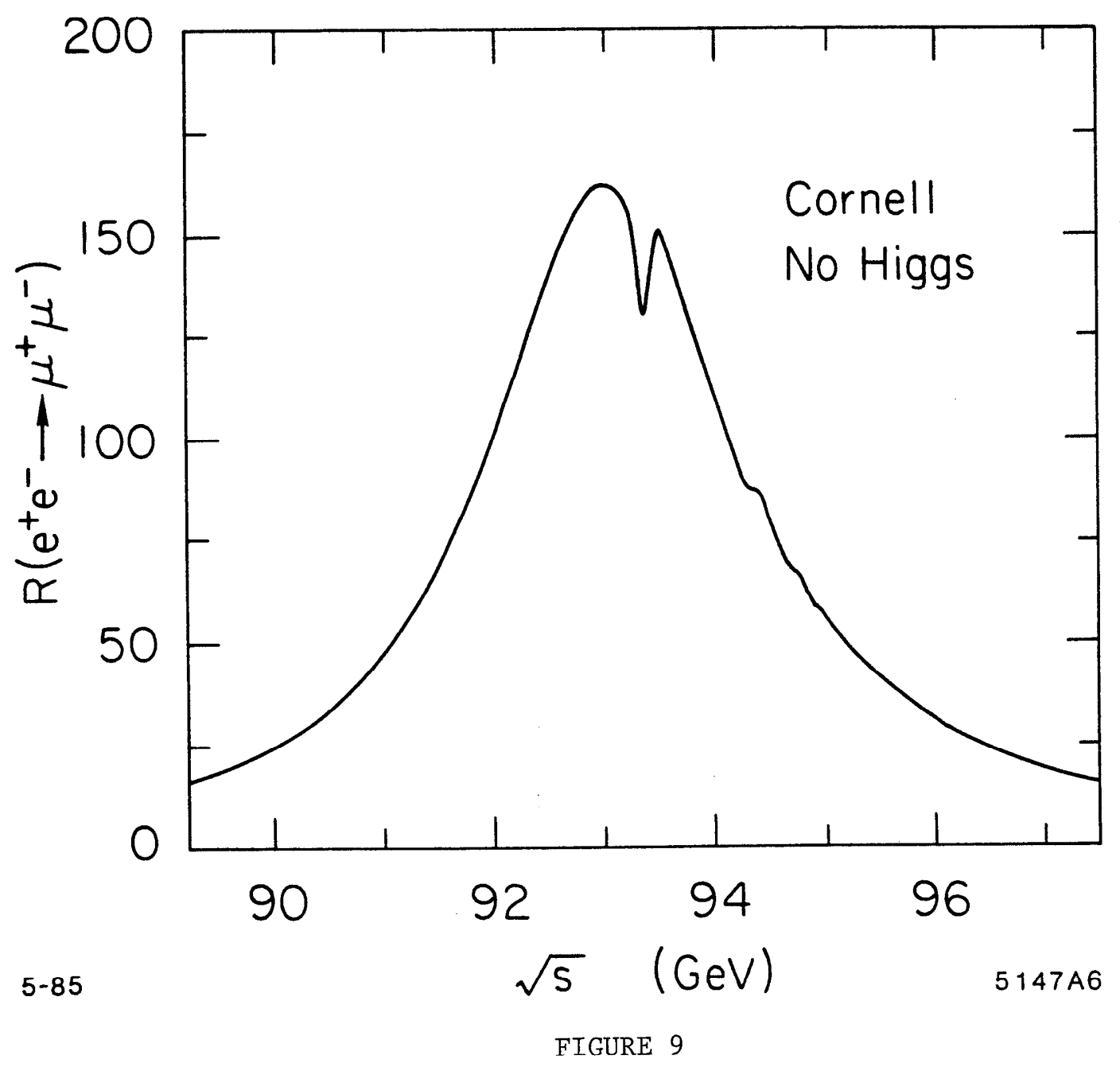




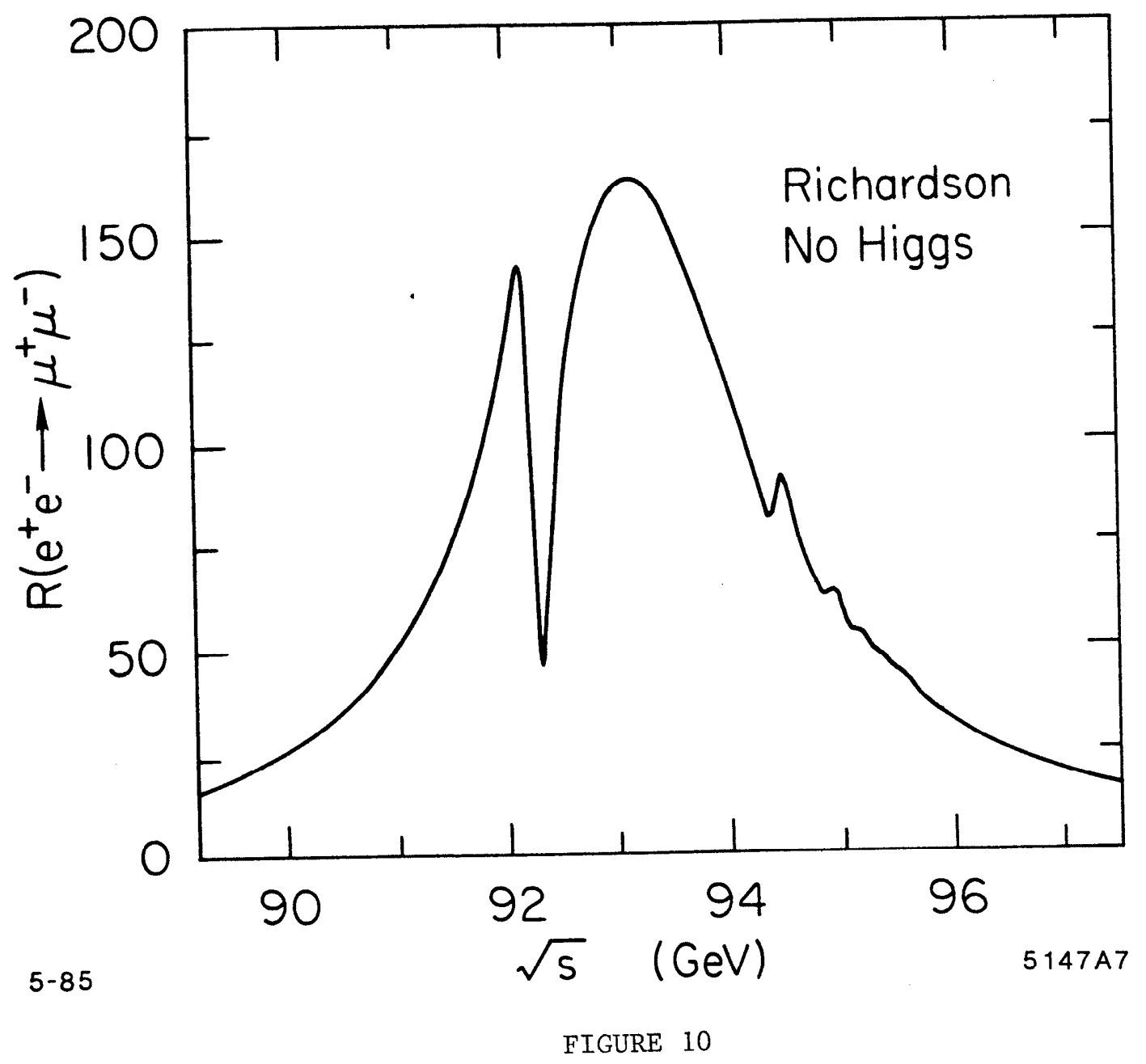




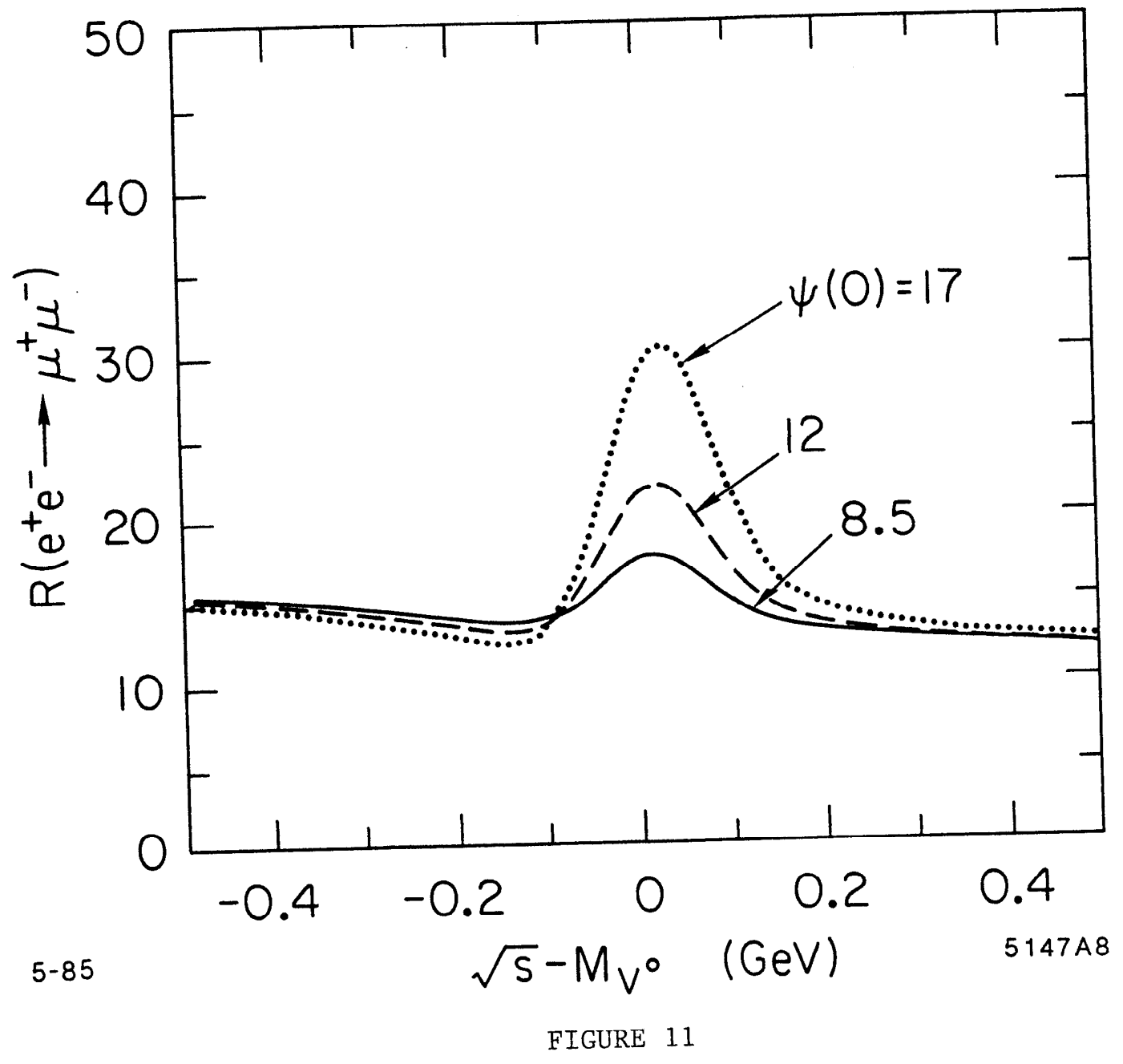




\section{PART III \\ CP VIOLATION AT THE UNIFICATION SCALE \\ Introduction}

It has recently been realized that a necessary and sufficient criterion for $\mathrm{CP}$ violation in the standard model with three generations can be formulated in a parametrization independent manner. This formulation is stated entirely in terms of the determinant of the commutator of the mass matrices for the charge $2 e / 3$ and charge $-e / 3$ quarks, ${ }^{[1]}$ a quantity invariant under any allowed redefinition of phases of the quark fields. It is not necessary to refer to the Kobayashi-Maskawa matrix, ${ }^{[2]}$ or any parametrization thereof. Instead one can work entirely with mass matrices which involve the fundamental Yukawa couplings of the Higgs boson(s) to the quarks in the basis of weak eigenstates.

With three generations of quarks and leptons CP is violated if and only if the single quantity,

$$
\operatorname{det} K=\operatorname{det}\left[U^{\dagger} U, D^{\dagger} D\right]
$$

is non-vanishing. Here $U$ and $D$ are the three by three Yukawa coupling matrices for the charge $2 e / 3$ and $-e / 3$ quarks, respectively.

In the Kobayashi-Maskawa ${ }^{[2]}$ parametrization

$$
\begin{aligned}
\operatorname{det} K \propto & \left(m_{t}^{2}-m_{c}^{2}\right)\left(m_{c}^{2}-m_{u}^{2}\right)\left(m_{u}^{2}-m_{t}^{2}\right)\left(m_{b}^{2}-m_{s}^{2}\right)\left(m_{a}^{2}-m_{d}^{2}\right)\left(m_{d}^{2}-m_{b}^{2}\right) \\
& \sin ^{2} \theta_{1} \sin \theta_{2} \sin \theta_{3} \cos \theta_{1} \cos \theta_{2} \cos \theta_{3} \sin \delta
\end{aligned}
$$

This vanishes if any quarks with the same charge have the same mass, or any of the angles $\theta_{i}$ assume the values 0 or $\pi / 2$, or the phase $\delta$ is 0 or $\pi$. 
In this paper we study the scale dependence of $\operatorname{det} K$. We find that it satisfies a simple renormalization group equation where the change in $\operatorname{det} K$ is proportional to itself. This allows a straightforward computation of its value at the grand unification scale in terms of its value at the weak scale, given an initial set of fermion masses and gauge couplings.

In the standard model with one Higgs doublet and three generations of quarks and leptons det $K$ falls by roughly six orders of magnitude in going from the weak to grand unification scales if the $t$ quark mass is small. When the $t$ quark mass approachs its fixed point value ${ }^{[4-\sigma]}$ of $\sim 220 \mathrm{GeV} / c^{2}$, $\operatorname{det} K$ falls by about four orders of magnitude. Similar results hold in extensions of the standard model involving two Higgs doublets or supersymmetry. The $\operatorname{decrease}$ in $\operatorname{det} K$ is due primarily to the decrease in quark masses that are not near their fixed points and has important consequences for baryogenesis.

\section{Renormalization Group Equation in the Standard Model}

The renormalization group equation for $\operatorname{det} K$ follows from those for the Yukawa coupling matrices $U$ and $D:^{[4-6]}$

$$
U^{-1} \frac{d U}{d \tau}=-G_{U}+3 T+\frac{3}{2}\left(U^{\dagger} U-D^{\dagger} D\right)
$$

and

$$
D^{-1} \frac{d D}{d \tau}=-G_{D}+3 T+\frac{3}{2}\left(D^{\dagger} D-U^{\dagger} U\right)
$$

The conventions are those of Ref. 6 , where $\tau=\frac{1}{16 \pi^{2}} \ln \left(\mu / M_{W}\right) ; T=T_{U}+$ $T_{D}=\operatorname{Tr}\left(U^{\dagger} U\right)+\operatorname{Tr}\left(D^{\dagger} D\right) ;$ and $G_{U}\left(G_{D}\right)$ is equal to $8 g_{3}^{2}+\frac{9}{4} g_{2}^{2}+\frac{17}{20} g_{1}^{2}$ and 
$8 g_{3}^{2}+\frac{9}{4} g_{2}^{2}+\frac{1}{4} g_{1}^{2}$, respectively. Leptons have been neglected as unimportant. Using Eqs. (3) and the definition of $K=\left[U^{\dagger} U, D^{\dagger} D\right]$, we find

$$
\frac{d K}{d \tau}=\{K, A\}
$$

where

$$
A=6 T+\frac{3}{2}\left(U^{\dagger} U+D^{\dagger} D\right)-\left(G_{U}+G_{D}\right)
$$

Noting that

$$
\frac{d(\ln \operatorname{det} K)}{d \tau}=\frac{d(\operatorname{Tr} \ln K)}{d \tau}=\operatorname{Tr}\left(K^{-1} \frac{d K}{d \tau}\right)=2 \operatorname{Tr} A
$$

we obtain:

$$
(\operatorname{det} K)_{\mu}=(\operatorname{det} K)_{M_{W}} e^{\int_{0}^{r}(2 \operatorname{TrA}) d \tau},
$$

where $\operatorname{Tr} A=\frac{39}{2} T-3\left(G_{U}+G_{D}\right)$.

The behavior of $\operatorname{det} K$ as a function of the momentum scale $\mu$ compared to its value at the weak scale is shown in Figure 1 for various values of $m_{t}$. The Yukawa couplings of the other quarks and the values of the gauge couplings are set at $M_{W}$ to their known values. ${ }^{[7]}$ It is seen that $\operatorname{det} K$ decreases as the scale $\mu$ increases. In particular, for $\mathrm{t}$ quark masses below about $150 \mathrm{GeV} / \mathrm{c}^{2},(\operatorname{det} K)_{\mu} /(\operatorname{det} K)_{M_{W}}$ decreases by over five orders of magnitude when $\mu$ is at the grand unification scale (which we take as $10^{15} \mathrm{GeV}$, corresponding to $\tau=0.19$ ). As $m_{t}$ grows larger, and the corresponding Yukawa coupling approaches its fixed point, ${ }^{[4-6]}$ $(\operatorname{det} K)_{G U T} /(\operatorname{det} K)_{W}$ approaches $\sim 10^{-4}$. 
Figure 2 shows the ratio of $\operatorname{det} K$ at the grand unification scale to its value at the weak scale as a function of $m_{t}$. Here we see more directly that $\operatorname{det} K$ at the unification scale assumes larger values as the t quark Yukawa coupling increases toward its fixed point. ${ }^{[1-6]}$

This already hints that most of the running of $\operatorname{det} K$ is due to the running of the quark masses rather than that of the mixing angles if we decompose $\operatorname{det} K$ into a product of factors, as in Eq. (2). The dashed curve in Figure 2, which shows only the effect of the running of the mass factors in Eq. (2), gives an explicit numerical demonstration that this is the case. An analytic calculation which neglects the contribution of the Yukawa couplings (a good approximation for small quark masses) shows the same result: most of the running is due to the quark masses, which fall by a factor of $\sim 3$ between the weak and grand unification scales.

With some hindsight, this is to be expected, as the mixing angles are dimensionless and functions of ratios of quark masses of the same charge. Thus they are insensitive to the running of the gauge couplings, which yield the same factor for all quarks of the same charge; the angles only run if there are large Yukawa couplings.

\section{Extension to Two Higgs Doublets and Supersymmetry}

To extend our results to the case with two Higgs doublets, we need only replace Eqs. (3) by

$$
U^{-1} \frac{d U}{d \tau}=-G_{U}+3 T_{U}+\frac{1}{2}\left(3 U^{\dagger} U+D^{\dagger} D\right)
$$


and

$$
D^{-1} \frac{d D}{d \tau}=-G_{D}+3 T_{D}+\frac{1}{2}\left(3 D^{\dagger} D+U^{\dagger} U\right)
$$

Correspondingly, the matrix A now is replaced by $A_{T w o}$ Higgs $_{s}=3 T+\frac{7}{2}\left(U^{\dagger} U+\right.$ $\left.D^{\dagger} D\right)-\left(G_{U}+G_{D}\right)$. The form of the remaining equations is the same, as is their solution. The only important difference is that there now exist two vacuum expectation values, $v_{u}$ and $v_{d}$, with the constraint that $v_{u}^{2}+v_{d}^{2}=v^{2}=(175 \mathrm{GeV})^{2}$. Thus in addition to $m_{t}$ we have an additional variable, which we take to be $v_{d}$.

In Figure 3 we plot the value of $\operatorname{det} K$ at the unification scale relative to its value at the weak scale as a function of $v_{d}$ for various values of $m_{t}$. Again, as in Figure 2, this ratio is roughly $10^{-6}$ when $m_{t}$ is small and grows to approximately $10^{-4}$ when the Yukawa coupling of the $t$ quark approaches its fixed point.

The situation for the supersymmetric extension of the standard model is similar. Now the Yukawa coupling matrices run according to

$$
U^{-1} \frac{d U}{d \tau}=-G_{U}+3 T_{U}+\left(3 U^{\dagger} U+D^{\dagger} D\right)
$$

and

$$
D^{-1} \frac{d D}{d \tau}=-G_{D}+3 T_{D}+\left(3 D^{\dagger} D+U^{\dagger} U\right)
$$

and $A_{S U S Y}=3 T+7\left(U^{\dagger} U+D^{\dagger} D\right)-\left(G_{U}+G_{D}\right)$. Because of contributions of super-partners, $G_{U}$ and $G_{D}$ are altered to $\frac{16}{3} g_{3}^{2}+3 g_{2}^{2}+\frac{13}{9} g_{1}^{2}$ and $\frac{16}{3} g_{3}^{2}+3 g_{2}^{2}+\frac{7}{9} g_{1}^{2}$, respectively. The behavior of $\operatorname{det} K$ is given in Figure 4, and is qualitatively the same as before. However, because the gauge couplings run more slowly, light quark masses decrease by a factor of $\sim 5$ rather than $\sim 3$ between the weak scale 
and the unification scale. Consequently $\operatorname{det} K$ at the unification scale is about three orders of magnitude smaller than for the standard model.

\section{Baryogenesis}

CP violation is one of the necessary conditions for baryogenesis. ${ }^{[8]}$ Conversely, baryogenesis is the only probe of the strength of CP violation at the grand unification scale.

A physical quantity involving CP violation does not have to be $\operatorname{det} K$ times pure numbers; it can involve det $K$ divided by other physical quantities such as quark masses, and therefore have a less dramatic decrease as we move from the weak to the unification scale. For example, analyses of baryon generation in a large class of theories ${ }^{[0]}$ lead to a baryon to photon ratio that scales like the product of six Yukawa couplings. ${ }^{[0]}$

If these Yukawa couplings are unrelated to quark masses, e.g., involve superheavy Higgs bosons that are not in the same $S U(5)$ multiplet as those that give masses to quarks, there is little predictive power. We fix our attention instead on theories where the baryon excess originates in the Yukawa couplings responsible for quark masses, and consequently can be obtained from accessible physics, say $\lesssim 10 \mathrm{TeV}$.

The standard cosmological scenario favors Higgs ${ }^{[11]}$ as a Baryon asymmetry * $(\Delta B)$ generator, since it may provide for the out of equilibrium decay; a necessary

$\star \Delta B$ is defined as the average baryon number per Higgs $(H)$ or anti-Higgs $(\bar{H})$ decay. If $r$ and $1-r$ are the branching ratios for $H \rightarrow q \ell$ and $H \rightarrow \overline{q q}$ and $\bar{r}$ and $1-\bar{r}$ are those for $H \rightarrow \bar{q} \bar{l}$ and $q q$, then

$$
\Delta B=\frac{1}{2}\left(\frac{1}{3} r-\frac{2}{3}(1-r)-\frac{1}{3} \bar{r}+\frac{2}{3}(1-\bar{r})\right)=\frac{1}{2}(r-\bar{r}) .
$$


condition on Baryon number violating processes in order to produce net baryon asymmetry.

The available couplings in minimal $S U(5)$ are shown in Figures 1 and 2 where $H, F_{L}$ subscripts stand for Higgs and left-handed fermions respectively. The numbers define the $S U(5)$ representations of the scalars and fermions.

Interference of tree graph Higgs and anti-Higgs boson decays to quarks results in $\Delta B=0$ because of CPT. The fourth order combination in Yukawa couplings of one tree and one loop diagrams also results.

$$
\Delta B \sim \operatorname{Im} \operatorname{Tr}\left(U^{+} U D^{+} D\right)=0
$$

The first non-trivial combination which results in $\Delta B \neq 0$ is given by a three loop diagram interferring with a tree $\operatorname{graph}^{[12]}$ as shown in Figure 3.

$$
\Delta B \sim \frac{I m T r\left[U^{+} D D^{+} U U^{+} U D^{+} D\right]}{16 \pi\left(8 \pi^{2}\right)^{2}\left[\operatorname{Tr}\left(U+U+D^{+} D\right)\right]}
$$

In the case of three generations with mixing and CP violating parameters in accordance with experimental constraints ${ }^{\dagger}$ we obtain $\Delta B_{W} \simeq 10^{-20}$. This is an unacceptably small value as the observed $\Delta B_{W} \simeq 10^{-9 \pm 1}$.

$\dagger \Delta B_{W, G}$ is the Baryon asymmetry at the weak scale and the GUT scale respectively.

$$
\begin{aligned}
m_{u} & =5 \times 10^{-3} \mathrm{GeV}, \quad m_{c}=1.25 \mathrm{GeV}, \quad m_{t}=50 \mathrm{GeV} \\
m_{d} & =9 \times 10^{-3} \mathrm{GeV}, \quad m_{s}=0.175 \mathrm{GeV}, \quad m_{b}=4.5 \mathrm{GeV} \\
V_{K M} & \equiv \Omega_{23} \Omega_{13} \Omega_{12} \quad[13]
\end{aligned}
$$

where

$$
\begin{aligned}
& \left(\Omega_{i j}\right)_{\alpha b}=0 \quad \text { for } \quad i, j \neq \alpha, b \\
& \left(\Omega_{i j}\right)_{i i}=\cos \phi_{i j}=\left(\Omega_{i j}\right)_{j j} \\
& \left(\Omega_{i j}\right)_{i j}=\sin \phi_{i j} \Delta_{i j}=-\left(\Omega_{i j}\right)_{j i}^{*}
\end{aligned}
$$


Four generations, with heavy masses and large mixings ${ }^{\ddagger}$ of the fourth generation result in a substantially bigger number, $\Delta B_{W} \sim 10^{-9}$ and $\Delta B_{G} \sim 10^{-11}$. Five generations easily give $\Delta B_{W} \sim 10^{-9}$ and $\Delta B_{G} \sim 10^{-10}$.

Improved experimental bounds of the mixing and $\mathrm{CP}$ violating parameters as well as on the heavy quark masses from forthcoming experiments will enable the sharper determination of $\Delta B$ in the future, within the context of theories examined here.

The number of light neutrino species-number of generations-extracted from the $Z^{0}$ width measurements at SLC will further constrain the above number

where

$$
\begin{gathered}
\Delta_{i j}= \begin{cases}1 & |i-j|=1 \\
e^{i \delta_{i j}} & |i-j|>1\end{cases} \\
U=\frac{1}{V} M_{U}=\frac{1}{V}\left(\begin{array}{ccc}
m_{u} & 0 & 0 \\
0 & m_{c} & 0 \\
0 & 0 & m_{t}
\end{array}\right) \quad(V=175 \mathrm{GeV}) \\
D=\frac{1}{V} M_{D}=\frac{1}{V} V_{K M}^{+}\left(\begin{array}{ccc}
m_{d} & 0 & 0 \\
0 & m_{\theta} & 0 \\
0 & 0 & m_{b}
\end{array}\right) V_{K M}
\end{gathered}
$$

With the above parameters we obtain

$$
\Delta B_{W}=1.7 \times 10^{-20} \text { and } \Delta B_{G}=10^{-23} .
$$

$\ddagger$ For four generations ${ }^{[13]}$

$$
V_{K M}=\Omega_{34} \Omega_{24} \Omega_{14} \Omega_{23} \Omega_{13} \Omega_{12}
$$

With the same quark masses and mixings for the first three generations, as in the previous footnote, and in addition

$$
\begin{aligned}
& S_{34}=0.22, \quad S_{24}=S_{14}=S_{13}=0.008 \\
& m_{T}=200 \mathrm{Gev}, \quad m_{B}=150 \mathrm{GeV}
\end{aligned}
$$

and $\delta_{24}=\delta_{14}=\delta_{19}=\pi / 4$, we obtain $\Delta B_{G} \simeq 10^{-11}$ and $\Delta B_{W} \simeq 10^{-10}$. 
for $\Delta B$.

Therefore, if the product of the six Yukawa couplings is proportional to light quark masses, one obtains too small a baryon excess. ${ }^{[12]}$ What we need are heavy generations of quarks whose masses are close to their fixed points and thus do not decrease as we go from the weak to grand unification scales. Their masses are constrained from above and below: if there exist $\mathrm{N}$ heavy generations then no quark can have a mass above the fixed point of $250 / \sqrt{N} \mathrm{GeV} / \mathrm{c}^{2}$ or else perturbative unification is lost..$^{|0|}$ If, on the other hand, they are much lighter than $250 / \sqrt{N} \mathrm{GeV} / \mathrm{c}^{2}$ they are too far from their fixed point; their masses will decrease as we go to the unification scale, and lead to too small a baryon excess.

We conclude in this framework that big bang baryogenesis suggests the existence of new heavy quarks close to their fixed points. Such quarks automatically occur $^{[14]}$ in family unified theories. ${ }^{[22,13]}$ 


\section{APPENDIX}

The evolution equation for the running coupling constant of $S U(3) \times S U(2) \times$ $U(1)$ is obtained as a solution to the following

$$
\frac{d g_{i}}{d t}=b_{i} g_{i}^{3}+\frac{1}{\left(16 \pi^{2}\right)} \sum_{j=1}^{3} b_{i j} g_{i}^{3} g_{j}^{2} \quad(j, i=1,2,3)
$$

Where $g_{1}, g_{2}, g_{3}$ are the coupling constants of $U_{1}, S U(2), S U(3)$ correspondingly. For the standard $S U(3) \times S U(2) \times U(1)$ model $b_{i}$ and $b_{i j}$ are given by

$$
\begin{aligned}
b_{i} & =\left(\begin{array}{c}
0 \\
-\frac{22}{3} \\
-11
\end{array}\right)+N_{g}\left(\begin{array}{c}
\frac{4}{3} \\
\frac{4}{3} \\
\frac{4}{3}
\end{array}\right)+H\left(\begin{array}{c}
\frac{1}{10} \\
\frac{1}{6} \\
0
\end{array}\right) \\
b_{i j} & =\left(\begin{array}{ccc}
0 & 0 & 0 \\
0 & -\frac{136}{3} & 0 \\
0 & 0 & -102
\end{array}\right)+N_{g}\left(\begin{array}{ccc}
\frac{19}{15} & \frac{3}{5} & \frac{44}{15} \\
\frac{1}{5} & \frac{49}{3} & 4 \\
\frac{11}{30} & \frac{3}{2} & \frac{76}{3}
\end{array}\right)+H\left(\begin{array}{ccc}
\frac{9}{50} & \frac{9}{10} & 0 \\
\frac{3}{10} & \frac{13}{6} & 0 \\
0 & 0 & 0
\end{array}\right)
\end{aligned}
$$

Where $N_{g}$ is the number of matter generations and $H$ is the number of Higgs doublets.

For the supersymmetric extension of the Standard Model the $b_{i}, b_{i j}$ are given by

$$
\begin{aligned}
& b_{i}=\left(\begin{array}{c}
0 \\
-6 \\
-9
\end{array}\right)+N_{g}\left(\begin{array}{l}
2 \\
2 \\
2
\end{array}\right)+H\left(\begin{array}{c}
\frac{3}{10} \\
\frac{1}{2} \\
0
\end{array}\right) \\
& b_{i j}=\left(\begin{array}{ccc}
0 & 0 & 0 \\
0 & -24 & 0 \\
0 & 0 & -54
\end{array}\right)+N_{g}\left(\begin{array}{ccc}
\frac{38}{15} & \frac{6}{5} & \frac{88}{15} \\
\frac{2}{5} & 14 & 8 \\
\frac{11}{15} & 3 & \frac{68}{3}
\end{array}\right)+H\left(\begin{array}{ccc}
\frac{9}{50} & \frac{9}{10} & 0 \\
\frac{3}{10} & \frac{7}{2} & 0 \\
0 & 0 & 0
\end{array}\right)
\end{aligned}
$$

Where $N_{g}$ is the number of generations of matter supermultiplets and $H$ is the number of Higgs doublet supermultiplets. 


\section{FIGURE CAPTIONS}

1) The (5)(5)(10) Yukawa coupling of minimal SU(5)

2) The (5)(10)(10) Yukawa coupling of minimal SU(5)

3) The lowest order Feynman Diagrams of minimal SU(5), whose interference results to a non zero Baryon Number

4) The ratio of $\operatorname{det} K$ at the momentum scale $\mu$ to its value at the weak scale plotted versus $\tau=\frac{1}{16 \pi^{2}} \ln \left(\mu / M_{W}\right)$ for various values of $m_{t}$.

5) The ratio (solid line) of $\operatorname{det} K$ at the unification scale $\left(\mu=10^{15} \mathrm{GeV}\right)$ to its value at the weak scale as a function of $m_{t}$. The dotted line shows this ratio due to the effect of the running of the mass factors in Eq. (2) alone.

6) The ratio of $\operatorname{det} K$ at the unification scale to its value at the weak scale in the case of two Higgs doublets as a function of $v_{d}$ for various values of $m_{t}$.

7) The ratio of $\operatorname{det} K$ at the unification scale to its value at the weak scale in the supersymmetric extension of the standard model as a function of $v_{d}$ for various values of $m_{t}$. 


\section{REFERENCES}

1. C. Jarlskog, Phys. Rev. Lett. $\underline{55}, 1039$ (1985); invited talk at the XXIth Rencontre de Moriond, March 9 - 16, 1986 and University of Stockholm preprint, 1986 (unpublished)

2. M. Kobayashi and T. Maskawa, Prog. Theor. Phys. $\underline{49}, 652$ (1973).

3. This differs from the quantity defined in Ref. 1 , which is not $\gamma_{5}$ invariant. $K$ contains the Yukawa couplings (which are dimensionless) rather than the masses, and $U^{\dagger} U$ and $D^{\dagger} D$, which are hermitian, rather than $U$ and $D$, which are generally not. The quantity in Ref. 1 and $\operatorname{det} K$ are equivalent as far as indicating CP violation if and only if they are non-vanishing.

4. B. Pendleton and G. Ross, Phys. Lett. 98B, 291 (1981); C. Hill, Phys. Rev. D24, 691 (1981).

5. E. Paschos, Z. Phys. $\underline{\text { C26 }}, 235$ (1984).

6. J. Bagger, S. Dimopoulos, and E. Masso, Nucl. Phys. B253, 397 (1985); Phys. Lett. 156B, 357 (1985); and Phys. Rev. Lett. $\underline{55}, 1450$ (1985).

7. The Yukawa coupling matrices at the weak scale are computed from the quark masses and the Kobayashi-Maskawa matrix with $\sin \theta_{1}=0.22$, $\sin \theta_{2}=0.03, \sin \theta_{3}=0.02$ and $\delta=\pi / 4$, which are values consistent with the measurements of the magnitudes of the Kobayashi-Maskawa matrix elements and with the magnitude of $\epsilon$ in the neutral K system. For the gauge couplings we use $g_{3}^{2}=1.70, g_{2}^{2}=0.45$, and $g_{1}^{2}=0.21$ and take $\Lambda_{\overline{M S}}=250 \mathrm{MeV}$. We have checked that our results for the running of 
$\operatorname{det} K$ are insensitive to the choice of the Kobayashi-Maskawa angles and to the values of the light quark masses.

8. A. D. Sakharov, Zh. Eksp. Teor. Fiz. Pisma $\underline{5}, 32$ (1967) [JETP Lett. $\underline{5}, 24$ (1967)]; M. Yoshimura, Phys. Rev. Lett. $\underline{41}, 281$ (1978); S. Dimopoulos and L. Susskind, Phys. Rev. D18, 4500 (1978); D. Toussaint, S. B. Treiman, F. Wilczek, and A. Zee, Phys. Rev. D19, 1036 (1979).

9. See, for example, the review by E. W. Kolb and M. S. Turner, Ann. Rev. Nucl. Sci. $\underline{33}, 645$ (1983) and references therein. We thank them for discussions on the history of these calculations.

10. In $\operatorname{det} K$ there are twelve powers of Yukawa couplings. In the analogous quantity defined in Ref. 1 there are six powers. Consequently, if we had chosen to consider the value of the latter quantity at the grand unification scale compared to its value at the weak scale, we would have found a decrease by about three orders of magnitude. Which quantity is relevant (if either is) depends on the physics of the mechanism of CP violation in the particular problem at hand.

11. M. Yoshimura, Phys. Lett. $\underline{88 \mathrm{~B}}$ (1979) 294; E. W. Kolb and S. Woldram, Nucl. Phys. B172 (1980) 224; J. N. Fry, K. Olive and M. S. Turner, Phys. Rev. D22 (1980) 2953, 2977.

12. J. Ellis, M. K. Gaillard, and D. V. Nanopoulos, Phys. Lett. $80 \mathrm{~B}, 360$ (1979) and $\underline{82 \mathrm{~B}}, 464$ (1979) (Erratum); D. V. Nanopoulos and S. Weinberg, Phys. Rev. D20, 2484 (1979); S. Barr, G. Segre, and H. A. Weldon, Phys. Rev. D20, 2494 (1979); G. Segre and M. S. Turner, Phys. Lett. $\underline{99 B}, 399$ (1981). 
13. H.Harari and M.Leurer, Phys. Lett. $\underline{181 \mathrm{~B}}, 123$ (1986).

14. J. Bagger and S. Dimopoulos, Nucl. Phys. B244, 247 (1984); J. Bagger, S. Dimopoulos, E. Masso, and M. H. Reno, Phys. Rev. Lett. 또, 2199 (1985); Nucl. Phys. B258, 565 (1985).

15. M. Gell-Mann, P. Ramond, and R. Slansky, in Supergravity, edited by P. van Nieuwenhuizen and D. Z. Freedman (North Holland, Amsterdam, 1979), p. 317; F. Wilczek and A. Zee, Phys. Rev. D25, 553 (1982). 


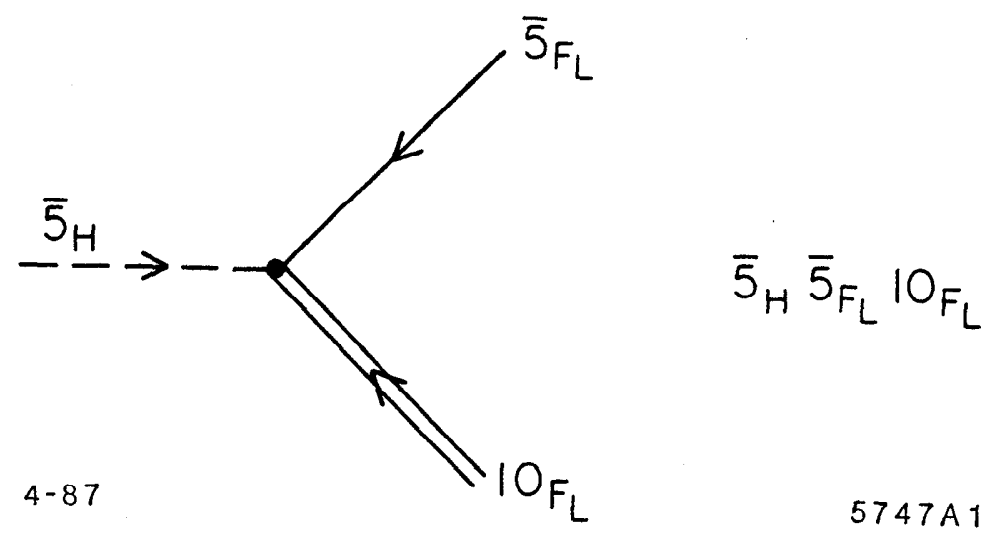

FIGURE 1 


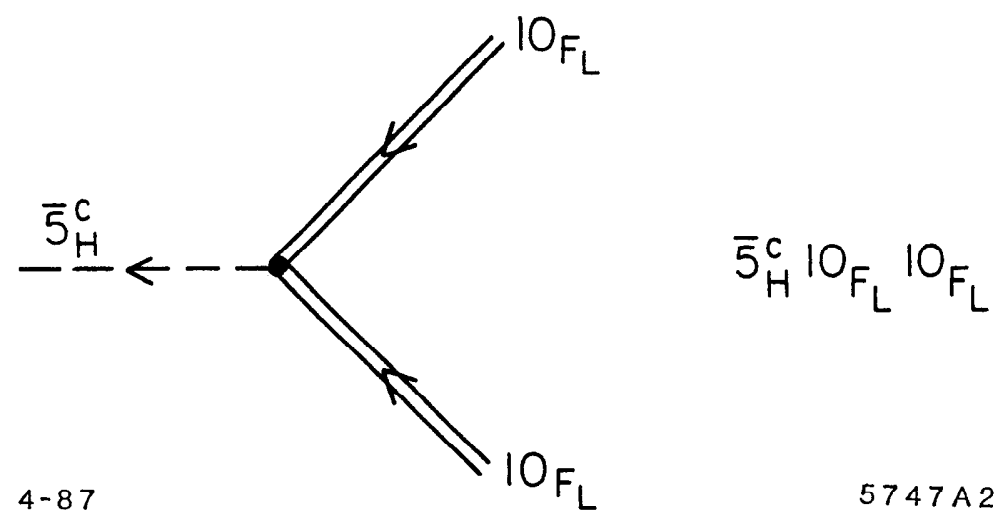

FTGURE 2 


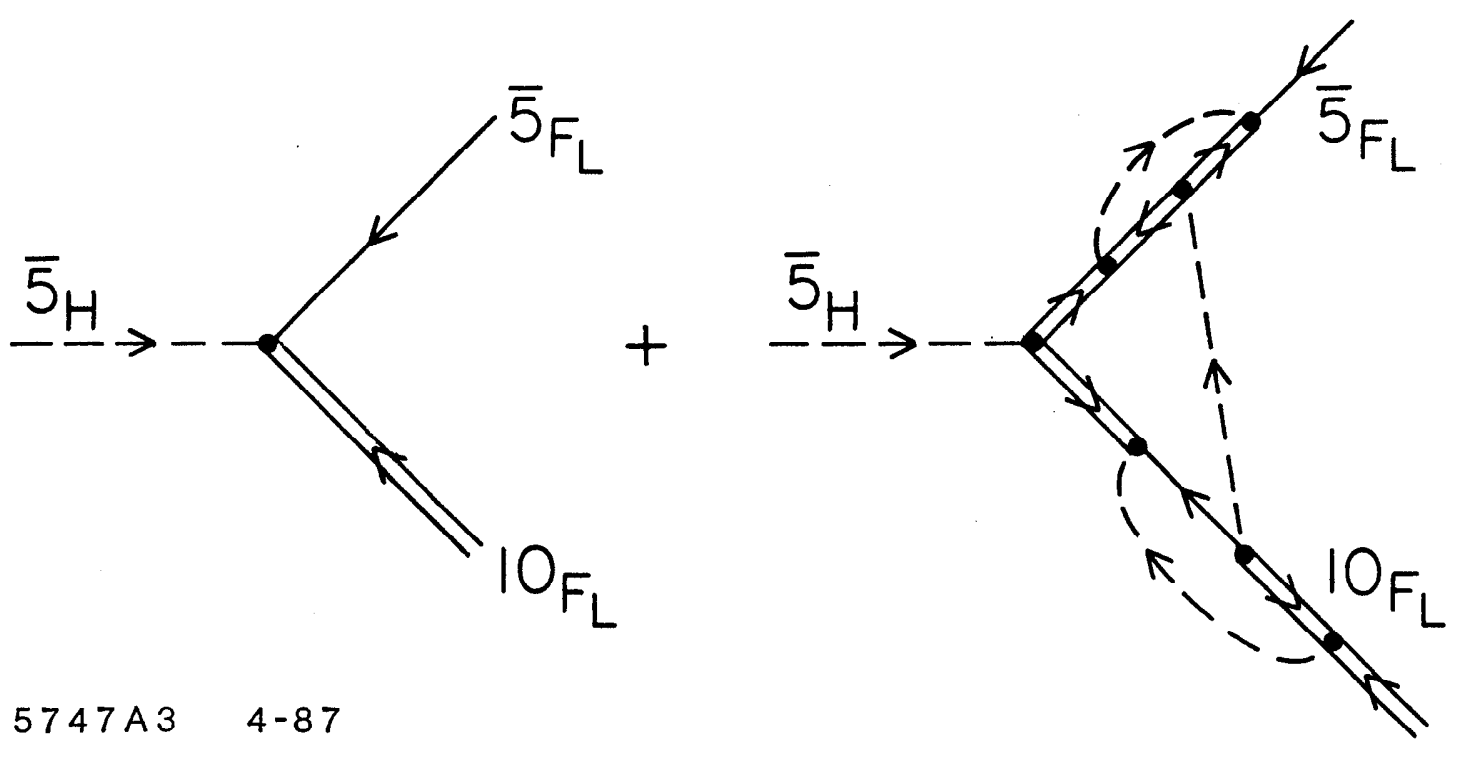

FIGURE 3 


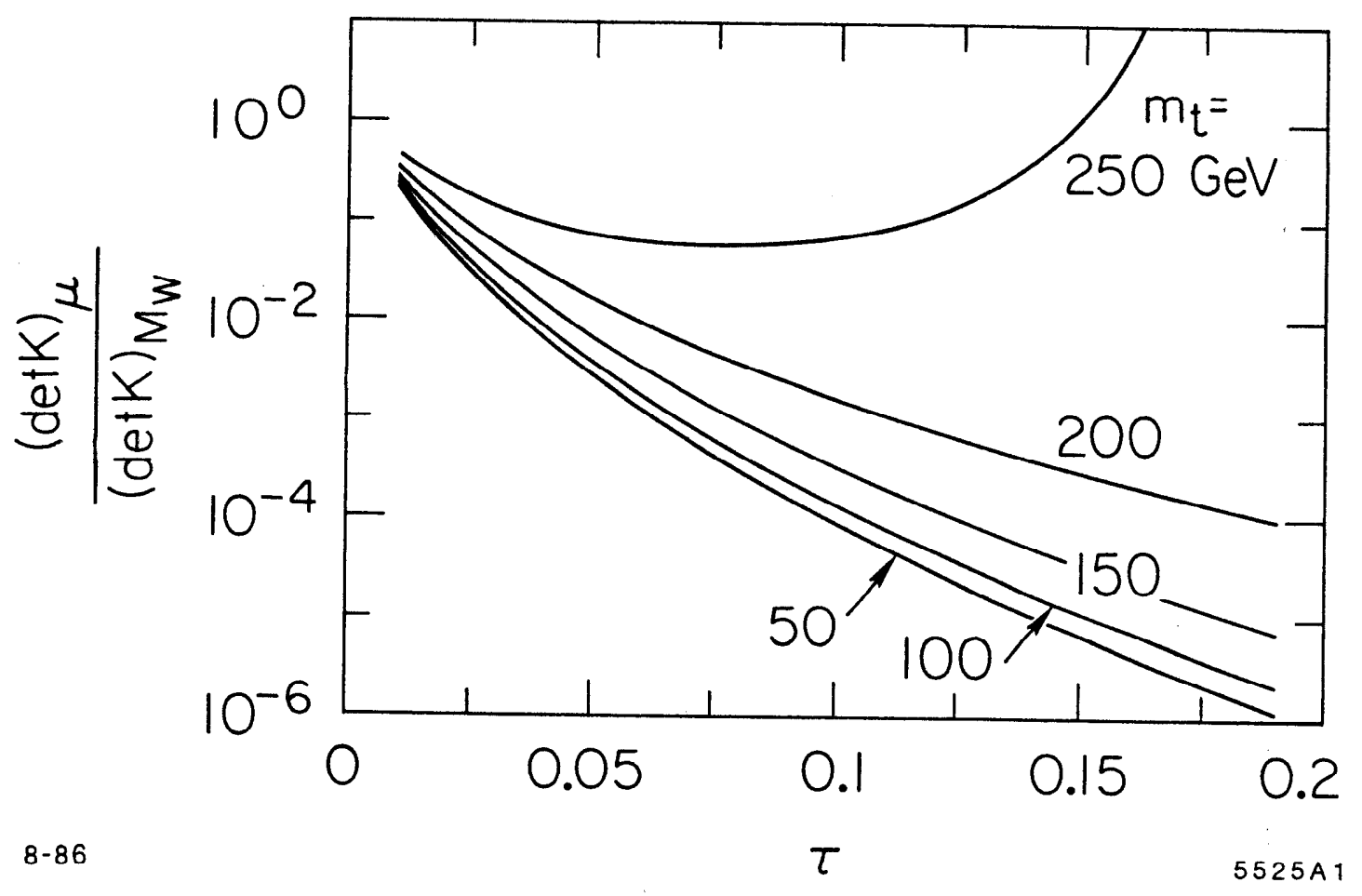

FIGURE 4 


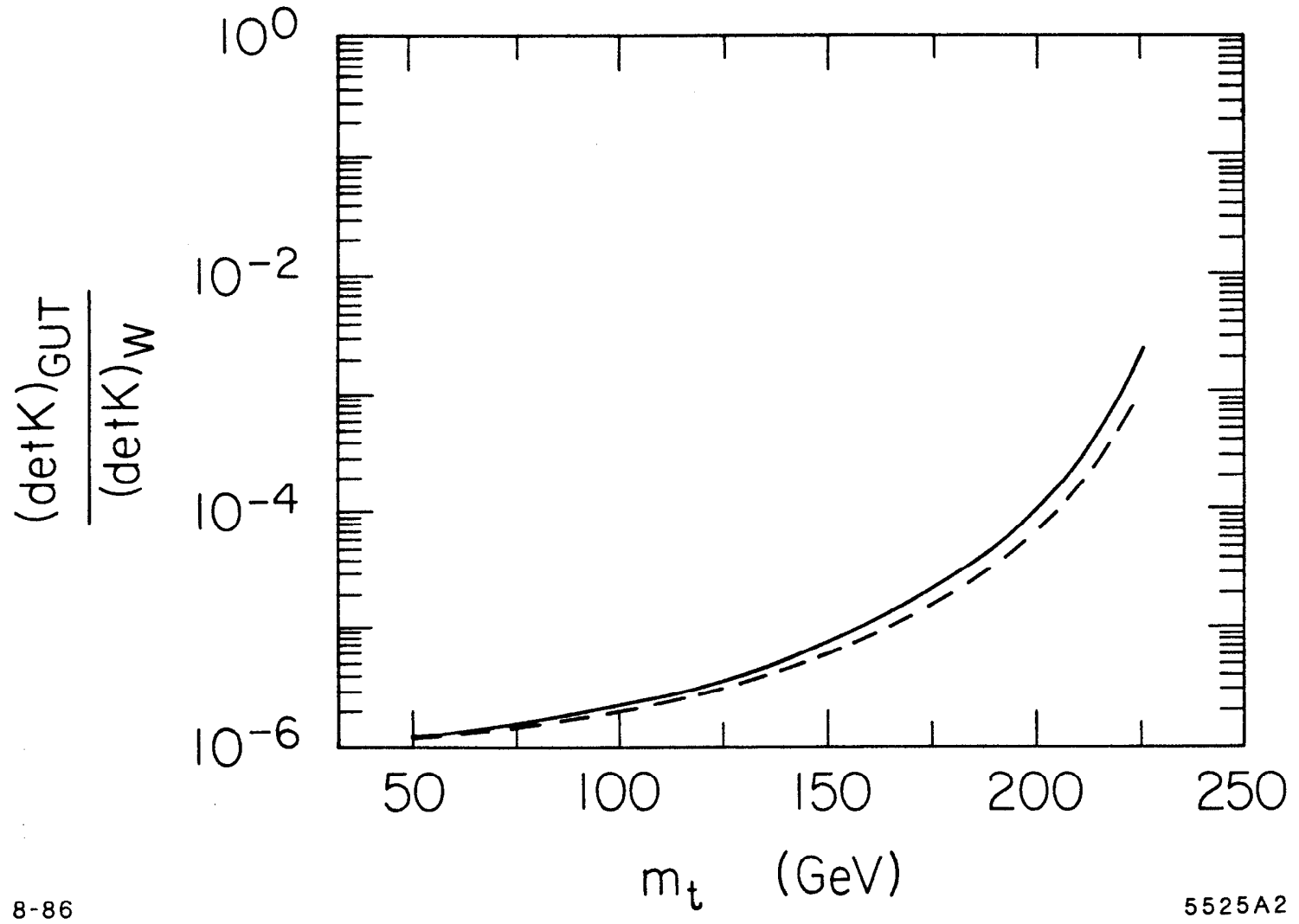

FIGURE 5 


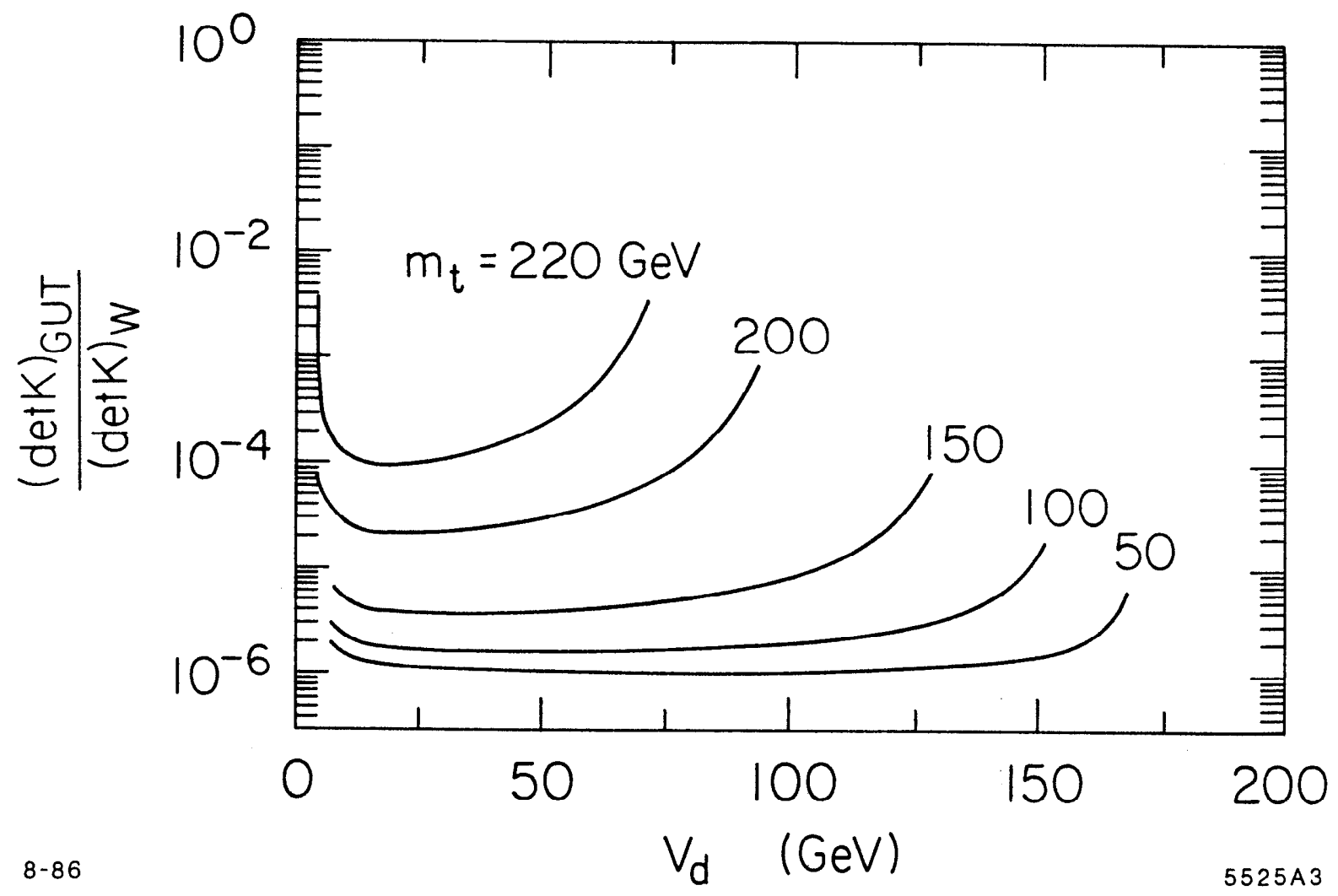

FIGURE 6 


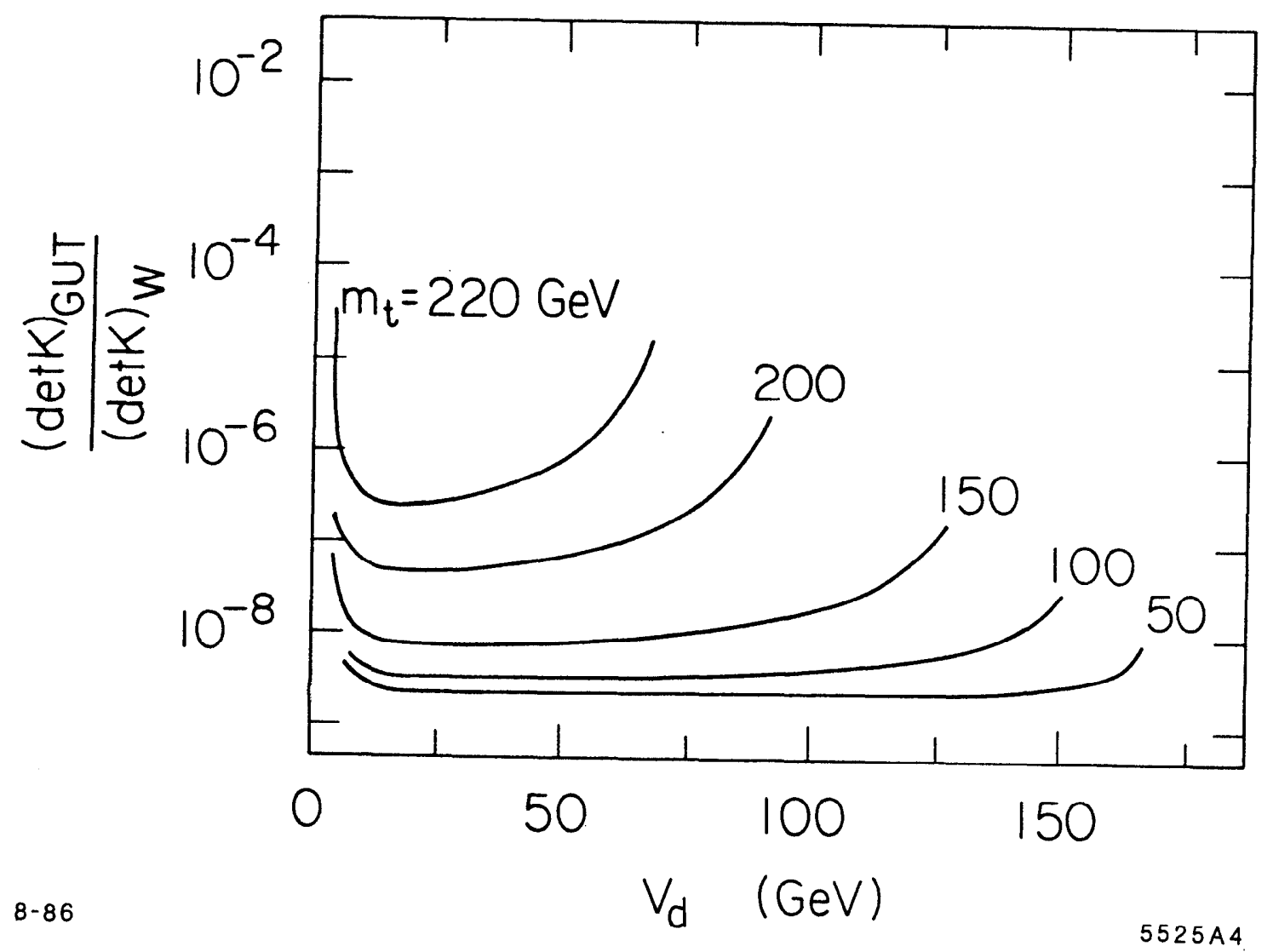

FIGURE 7 